\title{
Interacting Dark Matter and $q$-Deformed Dark Energy Nonminimally Coupled to Gravity
}

\author{
Emre Dil \\ Department of Physics, Sinop University, Korucuk, 57000 Sinop, Turkey \\ Correspondence should be addressed to Emre Dil; emredil@sakarya.edu.tr
}

Received 5 October 2016; Revised 10 November 2016; Accepted 17 November 2016

Academic Editor: Sergei D. Odintsov

Copyright ( 2016 Emre Dil. This is an open access article distributed under the Creative Commons Attribution License, which permits unrestricted use, distribution, and reproduction in any medium, provided the original work is properly cited. The publication of this article was funded by SCOAP .

\begin{abstract}
In this paper, we propose a new approach to study the dark sector of the universe by considering the dark energy as an emerging $q$-deformed bosonic scalar field which is not only interacting with the dark matter, but also nonminimally coupled to gravity, in the framework of standard Einsteinian gravity. In order to analyze the dynamic of the system, we first give the quantum field theoretical description of the $q$-deformed scalar field dark energy and then construct the action and the dynamical structure of this interacting and nonminimally coupled dark sector. As a second issue, we perform the phase-space analysis of the model to check the reliability of our proposal by searching the stable attractor solutions implying the late-time accelerating expansion phase of the universe.
\end{abstract}

\section{Introduction}

The dark energy is accepted as the effect of causing the latetime accelerated expansion of universe which is experienced by the astrophysical observations such as Supernova Ia $[1,2]$, large-scale structure [3, 4], the baryon acoustic oscillations [5], and cosmic microwave background radiation [6-9]. According to the standard model of cosmological data $70 \%$ of the content of the universe consists of dark energy. Moreover, the remaining $25 \%$ of the content is an unknown form of matter having a mass but in nonbaryonic form that is called dark matter and the other $5 \%$ of the energy content of the universe belongs to ordinary baryonic matter [10]. While the dark energy spread all over the intergalactic media of the universe and produces a gravitational repulsion by its negative pressure to drive the accelerating expansion of the universe, the dark matter is distributed over the inner galactic media inhomogeneously and it contributes to the total gravitational attraction of the galactic structure and fixes the estimated motion of galaxies and galactic rotation curves [11, 12].

Miscellaneous dark models have been proposed to explain a better mechanism for the accelerated expansion of the universe. These models include interactions between dark energy, dark matter, and the gravitational field. The coupling between dark energy and dark matter seems possible due to the equivalence of order of the magnitudes in the present time [13-22]. On the other hand, there are also models in which the dark energy nonminimally couples to gravity in order to provide quantum corrections and renormalizability of the scalar field in the curved spacetime. Also the crossing of the dark energy from the quintessence phase to phantom phase, known as the Quintom scenario, can be possible in the models where the dark energy interacts with the gravity. If the dark energy minimally couples to gravity, the equation of state parameter of the dark energy cannot cross the cosmological constant boundary $\omega=1$ in the Friedmann-RobertsonWalker (FRW) geometry; therefore it is possible to emerge the Quintom scenario in the model where the dark energy nonminimally couples to gravity [23-37].

The constitution of the dark energy can be alternatively the cosmological constant $\Lambda$ with a constant energy density filling the space homogeneously [38-41]. As the varying energy density dark energy models, instead of the cosmological constant, quintessence, phantom, and tachyon fields can be considered. However, all these different dark energy models are the same in terms of the nondeformed field constituting the dark energy. There is no reason to prevent us from assuming that the dark energy is a deformed scalar field, having a negative pressure, too, as expected from the dark energy. Therefore, we propose that the dark energy considered in 
this study is formed of the deformed scalar field whose field equations are defined by the deformed oscillator algebras.

The quantum algebra and quantum group structure were firstly introduced by Kulish et al. [42-44], during the investigations of integrable systems in quantum field theory and statistical mechanics. Quantum groups and deformed boson algebras are closely related terms. It is known that the deformation of the standard boson algebra is first proposed by Arik-Coon [45]. Later on, Macfarlane and Biedenharn have realized the deformation of boson algebra in a different manner from Arik-Coon [46, 47]. The relation between quantum groups and the deformed oscillator algebras can be constructed obviously with this study by expressing the deformed boson operators in terms of the $s u_{q}(2)$ Lie algebra operators. Therefore, the construction of the relation between quantum groups and deformed algebras leads the deformed algebras of great interest with many different applications. The deformed version of Bardeen-Cooper-Schrieffer (BCS) many-body formalism in nuclear force, deformed creation, and annihilation operators are used to study the quantum occupation probabilities [48]. As another study, in NambuJona-Lasinio (NJL) model, the deformed fermion operators are used instead of standard fermion operators and this leads to an increase in the NJL four-fermion coupling force and the quark condensation related to the dynamical mass [49]. The statistical mechanical studies of the deformed boson and fermion systems have been familiar in recent years [5060]. Moreover, the investigations on the internal structure of composite particles involve the deformed fermions or bosons as the building block of the composite structures [61, 62]. There are also applications of the deformed particles in black hole physics [63-66]. The range of the deformed boson and fermion applications diverses from atomic-molecular physics to solid state physics in a widespread manner [67-72].

The ideas on considering the dark energy as the deformed scalar field have become common in the literature [73-76]. In this study, we then take into account the deformed bosons as the scalar field dark energy interacting with the dark matter and also nonminimally coupled to gravity. In order to confirm our proposal that the dark energy can be considered as a deformed scalar field, we firstly introduce the dynamics of the interacting and nonminimally coupled dark energy, dark matter, and gravity model in a spatially flat FRW background and then perform the phase-space analysis to check whether it will provide the late-time stable attractor solutions implying the accelerated expansion phase of the universe.

\section{Dynamics of the Model}

The field equations of the scalar field dark energy are considered to be defined by the $q$-deformed boson fields in our model. Constructing a $q$-deformed quantum field theory after the idea of $q$-deformation of the single particle quantum mechanics [45-47] has naturally been nonsurprising [77-79]. The bosonic part of the deformed particle fields corresponds to the deformed scalar field and the fermionic counterpart corresponds to the deformed vector field. In this study, we consider the $q$-deformed bosonic scalar field as the $q$-deformed dark energy under consideration. In our model, the $q$ deformed dark energy interacts with the dark matter and also nonminimally couples to gravity.

Early Universe scenarios can be well understood by studying the quantum field theory in curved spacetime. The behavior of the classical scalar field near the initial singularity can be translated to the quantum field regime by constructing the coherent states in quantum mechanics for any mode of the scalar field. It is now impossible to determine the quantum state of the scalar field near the initial singularity by an observer, at the present universe. In order to overcome the undeterministic nature, Hawking proposes to take the random superposition of all possible states in that spacetime. It has been realized by Berger with taking random superposition of coherent states. Also the particle creation in an expanding universe with a nonquantized gravitational metric has been investigated by Parker. It has been stated by Goodison and Toms that if the field quanta obey the Bose or Fermi statistics, when considering the evolution of the scalar field in an expanding universe, then the particle creation does not occur in the vacuum state. Their result gives signification to the possibility of the existence of the deformed statistics in coherent or squeezed states in the Early Universe [79-84].

Motivated by this significant possibility, we propose that the dark energy consists of a $q$-deformed scalar field whose particles obey the $q$-deformed algebras. Therefore, we now define the $q$-deformed scalar field constructing the dark energy in our model. The field operator of the $q$-deformed scalar field dark energy can be given as [79]

$$
\phi_{q}(x)=\int \frac{d^{3} k}{(2 \pi)^{3 / 2}} \frac{1}{\left(2 w_{k}\right)^{1 / 2}}\left[a_{q}(k) e^{i k x}+a_{q}^{*}(k) e^{-i k x}\right] .
$$

The following commutation relations for the deformed annihilation operator $a_{q}(k)$ and creations operator $a_{q}^{*}(k)$ in $q^{-}$ bosonic Fock space are given by [45]

$$
\begin{aligned}
& a_{q}(k) a_{q}^{*}\left(k^{\prime}\right)-q^{2} a_{q}^{*}\left(k^{\prime}\right) a_{q}(k)=\delta\left(k-k^{\prime}\right), \\
& a_{q}(k) a_{q}\left(k^{\prime}\right)-q^{2} a_{q}\left(k^{\prime}\right) a_{q}(k)=0,
\end{aligned}
$$

where $q$ is a real deformation parameter in interval $0<q<\infty$ and $[\widehat{N}(k)]=a_{q}^{*}(k) a_{q}(k)$ is the deformed number operator of $k$ th mode whose eigenvalue spectrum is given as

$$
[N(k)]=\frac{1-q^{2 N(k)}}{1-q^{2}} .
$$

Here $\widehat{N}(k)=a_{s}^{*}(k) a_{s}(k)$ is the standard nondeformed number operator. By using (2) in (1), we can obtain the commutation relations and planewave expansion of the $q$-deformed scalar field $\phi_{q}(x)$, as follows:

$$
\phi_{q}(x) \phi_{q}^{*}\left(x^{\prime}\right)-q^{2} \phi_{q}^{*}\left(x^{\prime}\right) \phi_{q}(x)=i \Delta\left(x-x^{\prime}\right),
$$

where

$$
\Delta\left(x-x^{\prime}\right)=\frac{-1}{(2 \pi)^{3}} \int \frac{d^{3} k}{w_{k}} \sin w_{k}\left(x-x_{0}\right) .
$$


The metric of the spatially flat FRW spacetime in which the $q$ oscillator algebra represents the $q$-deformed scalar field dark energy is defined by

$$
d s^{2}=-d t^{2}+a^{2}(t)\left[d r^{2}+r^{2} d \theta^{2}+r^{2} \sin ^{2} \theta d \phi^{2}\right],
$$

and for a FRW metric

$$
w_{k}^{2}=g\left(\sum_{i} \frac{k_{i}^{2}}{a^{2}}+m\right),
$$

where $g=\operatorname{det} g_{\mu \nu}$. Also the relation between deformed and standard annihilation operators $a_{q}$ and $a_{s}$ [85] is given as

$$
a_{q}=a_{s} \sqrt{\frac{[\widehat{N}]}{\widehat{N}}},
$$

which is used to obtain the relation between deformed and standard bosonic scalar fields by using (3) in (8) and (1):

$$
\phi_{q}=\phi \sqrt{\frac{1-q^{2 \widehat{N}}}{\left(1-q^{2}\right) \widehat{N}}} .
$$

Here we have used the Hermiticity of the number operator $\widehat{N}$.

Now the Friedmann equations will be derived for our interacting dark matter and nonminimally coupled $q$ deformed dark energy model in a FRW spacetime by using the scale factor $a(t)$ in Einstein's equations. In order to obtain these equations, we relate the scale factor to the energymomentum tensor of the objects in the model under consideration. We use the fluid description of the objects in our model by considering energy and matter as a perfect fluid, which are dark energy and matter in our model. An isotropic fluid in one coordinate frame leads to an isotropic metric in another frame coinciding with the frame of the fluid. This means that the fluid is at rest in commoving coordinates. Then the four velocities of the fluid are given as [52]

$$
U^{\mu}=(1,0,0,0),
$$

and the energy-momentum tensor follows as

$$
T_{\mu \nu}=(\rho+p) U_{\mu} U_{\nu}+p g_{\mu \nu}=\left(\begin{array}{cccc}
\rho & 0 & 0 & 0 \\
0 & & \\
0 & g_{i j} p \\
0 &
\end{array}\right)
$$

A more suitable form can be obtained by raising one, such that

$$
T_{v}^{\mu}=\operatorname{diag}(-\rho, p, p, p)
$$

Since we have two constituents, $q$-deformed dark energy and the dark matter in our model, the total energy density and the pressure are given by

$$
\begin{aligned}
& \rho_{\text {tot }}=\rho_{q}+\rho_{m}, \\
& p_{\text {tot }}=p_{q}+p_{m},
\end{aligned}
$$

where $\rho_{q}$ and $p_{q}$ are the energy density and the pressure of the $q$-deformed dark energy and $\rho_{m}$ and $p_{m}$ are the energy density and the pressure of the dark matter, respectively. The equation of state of the energy-momentum carrying cosmological fluid component under consideration in the FRW universe is given by $p=\omega \rho$ which relates the pressure and the energy density and $\omega$ is called the equation of state parameter. We then express the total equation of state parameter, such that

$$
\omega_{\text {tot }}=\frac{p_{\text {tot }}}{\rho_{\text {tot }}}=\omega_{q} \Omega_{q}+\omega_{m} \Omega_{m}
$$

where $\Omega_{q}=\rho_{q} / \rho_{\text {tot }}$ and $\Omega_{m}=\rho_{m} / \rho_{\text {tot }}$ are the density parameters for the $q$-deformed dark energy and the dark matter, respectively. Then the total density parameter is defined as

$$
\Omega_{\mathrm{tot}}=\Omega_{q}+\Omega_{m}=\frac{\kappa^{2} \rho_{\mathrm{tot}}}{3 H^{2}}=1
$$

We now turn to Einstein's equations of the form $R_{\mu \nu}=$ $\kappa^{2}\left(T_{\mu \nu}-(1 / 2) g_{\mu \nu} T\right)$. Then, by using the components of the Ricci tensor for a FRW spacetime (6) and the energy-momentum tensor in (12), we rewrite Einstein's equations, for $\mu \nu=00$ and $\mu \nu=i j$, as follows:

$$
\begin{aligned}
-3 \frac{\ddot{a}}{a} & =\frac{\kappa^{2}}{2}(\rho+3 p), \\
\frac{\ddot{a}}{a}+2\left(\frac{\dot{a}}{a}\right)^{2} & =\frac{\kappa^{2}}{2}(\rho-p),
\end{aligned}
$$

respectively. Here dot also represents the derivative with respect to cosmic time $t$. Using (16) and (17) gives the Friedmann equations for the FRW metric as

$$
\begin{aligned}
H^{2} & =\frac{\kappa^{2}}{3}\left(\rho_{q}+\rho_{m}\right), \\
\dot{H} & =-\frac{\kappa^{2}}{2}\left(\rho_{q}+p_{q}+\rho_{m}+p_{m}\right),
\end{aligned}
$$

where $H=\dot{a} / a$ is the Hubble parameter. From the conservation of energy, we can obtain the continuity equations for the $q$-deformed dark energy and the dark matter constituents in the form of evolution equations, such as

$$
\begin{gathered}
\dot{\rho}_{q}+3 H\left(\rho_{q}+p_{q}\right)=-Q, \\
\dot{\rho}_{m}+3 H\left(\rho_{m}+p_{m}\right)=Q,
\end{gathered}
$$

where $Q$ is an interaction current between the $q$-deformed dark energy and the dark matter which transfers the energy and momentum from the dark matter to dark energy and vice versa. $Q$ vanishes for the models having no interaction between the dark energy and the dark matter.

Now we will define the Dirac-Born-Infeld type action integral of the interacting dark matter and $q$-deformed dark energy nonminimally coupled to gravity in the framework of Eisteinian general relativity [86-88]. After that we will obtain the energy-momentum tensor $T_{\mu \nu}$ for the $q$-deformed dark 
energy and the dark matter in order to get the energy density $\rho$ and pressure $p$ of these dark objects explicitly. Then the action is given as

$$
\begin{aligned}
S= & \int d^{4} x \sqrt{-g}\left[\frac{R}{2 \kappa^{2}}-\frac{1}{2} g^{\mu \nu} \partial_{\mu} \phi_{q} \partial_{\nu} \phi_{q}-V\right. \\
& \left.-\xi f\left(\phi_{q}\right) R+L_{m}\right],
\end{aligned}
$$

where $\xi$ is a dimensionless coupling constant between $q$ deformed dark energy and the gravity, so $\xi f\left(\phi_{q}\right) R$ denotes the explicit nonminimal coupling between energy and the gravity. Also $L_{q}=-(1 / 2) g^{\mu \nu} \partial_{\mu} \phi_{q} \partial_{\nu} \phi_{q}-V-\xi f\left(\phi_{q}\right) R$ and $L_{m}$ are the Lagrangian densities of the $q$-deformed dark energy and the dark matter, respectively. Then the energymomentum tensors of the dark energy constituent of our model can be calculated, as follows [89]:

$$
\begin{aligned}
T_{\mu \nu}^{q}= & -2 \frac{\partial L_{q}}{\partial g^{\mu \nu}}+g_{\mu \nu} L_{q} \\
= & \partial_{\mu} \phi_{q} \partial_{\nu} \phi_{q}+2 \xi f\left(\phi_{q}\right) \frac{\partial R}{\partial g^{\mu \nu}} \\
& -\frac{1}{2} g_{\mu \nu}\left[g^{\alpha \beta} \partial_{\alpha} \phi_{q} \partial_{\beta} \phi_{q}+2 V\right]-g_{\mu \nu} \xi f\left(\phi_{q}\right) R .
\end{aligned}
$$

In order to find the derivative of the Ricci scalar with respect to the metric tensor, we use the variation of the contraction of the Ricci tensor identity $\delta R=R_{\mu \nu} \delta g^{\mu \nu}+g^{\mu \nu} \delta R_{\mu \nu}$. This leads us to finding the variation of the contraction of the Riemann tensor identity, as follows: $\delta R_{\mu \nu}=\delta R_{\mu \rho \nu}^{\rho}=\nabla_{\rho}\left(\delta \Gamma_{\nu \mu}^{\rho}\right)-$ $\nabla_{\nu}\left(\delta \Gamma_{\rho \mu}^{\rho}\right)$. Here $\nabla_{\mu}$ represents the covariant derivative and $\Gamma_{\nu \mu}^{\rho}$ represents the Christoffel connection. By using the metric compatibility and the tensor nature of $\delta \Gamma_{\nu \mu}^{\rho}$, we finally obtain

$$
\frac{\delta R}{\delta g^{\mu \nu}}=R_{\mu \nu}+g_{\mu \nu} \square-\nabla_{\mu} \nabla_{\nu},
$$

where $\square=g^{\alpha \beta} \nabla_{\alpha} \nabla_{\beta}$ is the covariant d'Alembertian. Using (23) in (22) gives

$$
\begin{aligned}
T_{\mu \nu}^{q}= & \partial_{\mu} \phi_{q} \partial_{\nu} \phi_{q}-\frac{1}{2} g_{\mu \nu}\left[g^{\alpha \beta} \partial_{\alpha} \phi_{q} \partial_{\beta} \phi_{q}\right]-g_{\mu \nu} V \\
& +2 \xi\left[R_{\mu \nu}-\frac{1}{2} g_{\mu \nu} R\right] f\left(\phi_{q}\right)+2 \xi \square f\left(\phi_{q}\right) \\
& -2 \xi \nabla_{\mu} \nabla_{\nu} f\left(\phi_{q}\right) .
\end{aligned}
$$

Then the $\mu \nu=0,0$ component of the energy-momentum tensor leads to the energy density $\rho_{q}$ :

$$
\rho_{q}=T_{00}^{q}=\frac{1}{2} \dot{\phi}_{q}^{2}+V+6 \xi H^{2} f\left(\phi_{q}\right)+6 \xi H f^{\prime}\left(\phi_{q}\right) \dot{\phi}_{q}
$$

where prime refers to derivative with respect to the field $\phi_{q}$ and we use $\square=-\partial_{0}^{2}-3 H \partial_{0}$, because of the homogeneity and the isotropy for $\phi_{q}$ in space. Also $R_{00}=-3 \ddot{a} / a$ and
$R=6\left[\ddot{a} / a+\dot{a}^{2} / a^{2}\right]$ is used for the FRW geometry. The $\mu \nu=i, i$ components of $T_{\mu \nu}^{q}$ also give the pressure $p_{q}$ as

$$
\begin{aligned}
p_{q} & =g^{i i} T_{i i}^{q}=\frac{1}{2} \dot{\phi}_{q}^{2}-V-2 \xi\left[2 \dot{H} f\left(\phi_{q}\right)+3 H^{2} f\left(\phi_{q}\right)\right. \\
& \left.+f^{\prime \prime}\left(\phi_{q}\right) \dot{\phi}_{q}^{2}+f^{\prime}\left(\phi_{q}\right) \ddot{\phi}_{q}+2 H f^{\prime}\left(\phi_{q}\right) \dot{\phi}_{q}\right]
\end{aligned}
$$

where we use $\left(\nabla_{i} \nabla_{i}\right) f\left(\phi_{q}\right)=\left(\partial_{i} \nabla_{i}-\Gamma_{i i}^{\lambda} \nabla_{\lambda}\right) f\left(\phi_{q}\right)=-\Gamma_{11}^{0} \partial_{0} f\left(\phi_{q}\right)$ with $\Gamma_{11}^{0}=\dot{a} a$ for the FRW spacetime. We can now obtain the equation of motion for the $q$-deformed dark energy by inserting (25) and (26) into the evolution equation (19), such that

$$
\ddot{\phi}_{q}+3 H \dot{\phi}_{q}+\frac{\partial V}{\partial \phi_{q}}+\xi R f^{\prime}\left(\phi_{q}\right)=-\frac{Q}{\dot{\phi}_{q}} .
$$

The usual assumption in the literature is to consider the coupling function as $f\left(\phi_{q}\right)=\phi_{q}^{2} / 2$ [90] and the potential as $V=$ $V_{0} e^{-\kappa \lambda \phi_{q}}$ [91-93]. In order to find the energy density, pressure, and equation of motion in terms of the deformation parameter $q$, we use the above coupling function and potential with the rearrangement of equation (9) as $\phi_{q}=\Delta(q) \phi$ in the equations (25)-(27) and obtain

$$
\begin{aligned}
\rho_{q} & =\frac{1}{2} \Delta^{2} \dot{\phi}^{2}+e^{-\kappa \lambda \Delta \phi}+3 \xi H^{2} \Delta^{2} \phi^{2}+6 \xi H \Delta^{2} \phi \dot{\phi} \\
& +\frac{1}{2} \dot{\Delta}^{2} \phi^{2}+\Delta \dot{\Delta} \phi \dot{\phi}+6 \xi H \Delta \dot{\Delta} \phi^{2}, \\
p_{q} & =\frac{1}{2} \Delta^{2} \dot{\phi}^{2}-e^{-\kappa \lambda \Delta \phi} \\
& -2 \xi \Delta^{2}\left[\dot{H} \phi^{2}+\frac{3}{2} H^{2} \phi^{2}+\dot{\phi}^{2}+\phi \ddot{\phi}+2 H \phi \dot{\phi}\right] \\
& +(1-8 \xi) \Delta \dot{\Delta} \phi \dot{\phi}+\left(\frac{1}{2}-2 \xi\right) \Delta^{2} \phi^{2}-2 \xi \Delta \ddot{\Delta} \phi^{2} \\
& -4 \xi H \phi \dot{\phi} \Delta \dot{\Delta} \phi^{2}, \\
\Delta \ddot{\phi} & +3 \Delta H \dot{\phi}-\kappa \lambda e^{-\kappa \lambda \Delta \phi}+\xi \Delta R \dot{\phi}+2 \dot{\Delta} \dot{\phi}+\ddot{\Delta} \phi \\
& +3 H \dot{\Delta} \phi=-\beta \kappa \rho_{m} .
\end{aligned}
$$

Here we consider that the particles in each mode can vary by creation or annihilation in time for $\Delta=\sqrt{\left(1-q^{2 N}\right) /\left(1-q^{2}\right) N}$; therefore its time derivatives are nonvanishing. On the other hand, the common interaction current in the literature $Q=\beta \kappa \rho_{m} \dot{\phi}_{q}$ is used here [17].

Now the phase-space analysis for our interacting dark matter and nonminimally coupled $q$-deformed dark energy model will be performed, whether the late-time stable attractor solutions can be obtained, in order to confirm our model.

\section{Phase-Space and Stability Analysis}

The cosmological properties of the proposed $q$-deformed dark energy model can be investigated by performing the 
phase-space analysis. Therefore, we first transform the equations of the dynamical system into its autonomous form by introducing the auxiliary variables $[15,94-98]$, such as

$$
\begin{aligned}
& x=\frac{\kappa \Delta \dot{\phi}}{\sqrt{6} H}=\Delta x_{s}, \\
& y=\frac{\kappa \sqrt{e^{-\kappa \lambda \Delta \phi}}}{\sqrt{3} H}=\sqrt{e^{-\kappa \lambda \phi(\Delta-1)}} y_{s}, \\
& z=\frac{\kappa \dot{\Delta} \phi}{\sqrt{6} H}, \quad z_{s}=0 \\
& u=\kappa \Delta \phi=\Delta u_{s},
\end{aligned}
$$

where $x_{s}, y_{s}, z_{s}$, and $u_{s}$ are the standard form of the auxiliary variables in $q \rightarrow 1$ limit. We now write the density parameters for the dark matter and $q$-deformed scalar field dark energy in the autonomous system by using (28) with (36):

$$
\begin{aligned}
\Omega_{m}= & \frac{\kappa^{2} \rho_{m}}{3 H^{2}}, \\
\Omega_{q}= & \frac{\kappa^{2} \rho_{q}}{3 H^{2}} \\
= & x^{2}+y^{2}+\xi u^{2}+2 \sqrt{6} \xi x u+z^{2}+2 x z \\
& +2 \sqrt{6} \xi z u .
\end{aligned}
$$

Then the total density parameter reads

$$
\begin{aligned}
\Omega_{\mathrm{tot}}= & \frac{\kappa^{2} \rho_{\mathrm{tot}}}{3 H^{2}} \\
= & x^{2}+y^{2}+\xi u^{2}+2 \sqrt{6} \xi x u+z^{2}+2 x z \\
& +2 \sqrt{6} \xi z u+\Omega_{m}=1 .
\end{aligned}
$$

We should also obtain the $\kappa^{2} p_{q} / 3 H^{2}$ in the autonomous form to write the equation of state parameters, such that

$$
\begin{aligned}
\frac{\kappa^{2} p_{q}}{3 H^{2}}= & (1-4 \xi) x^{2}-y^{2}+\left(\frac{2}{3} \xi+4 \xi^{2}\right) s u^{2} \\
& +\left(8 \xi^{2}-\xi\right) u^{2}+\frac{2 \sqrt{6}}{3} \xi x u+(1-4 \xi) z^{2} \\
& +2(1-4 \xi) x z+\frac{2 \sqrt{6}}{3} \xi z u+2 \xi \beta u \Omega_{m} \\
& -2 \xi \lambda y^{2} u,
\end{aligned}
$$

where $s=-\dot{H} / H^{2}$. Using (33) and (35), we find the equation of state parameter for the dark energy as

$$
\begin{aligned}
\omega_{q} & =\frac{p_{q}}{\rho_{q}}=\left[(1-4 \xi) x^{2}-y^{2}+\left(\frac{2}{3} \xi+4 \xi^{2}\right) s u^{2}\right. \\
& +\left(8 \xi^{2}-\xi\right) u^{2}+\frac{2 \sqrt{6}}{3} \xi x u+(1-4 \xi) z^{2}
\end{aligned}
$$

$$
\begin{aligned}
& \left.+2(1-4 \xi) x z+\frac{2 \sqrt{6}}{3} \xi z u+2 \xi \beta u \Omega_{m}-2 \xi \lambda y^{2} u\right] \\
& \cdot\left[x^{2}+y^{2}+\xi u^{2}+2 \sqrt{6} \xi x u+z^{2}+2 x z\right. \\
& +2 \sqrt{6} \xi z u]^{-1} .
\end{aligned}
$$

Also from (33) and (36), the total equation of state parameter can be obtained as

$$
\begin{aligned}
\omega_{\text {tot }}= & \omega_{q} \Omega_{q}+\omega_{m} \Omega_{m} \\
= & (1-4 \xi) x^{2}-y^{2}+\left(\frac{2}{3} \xi+4 \xi^{2}\right) s u^{2} \\
& +\left(8 \xi^{2}-\xi\right) u^{2}+\frac{2 \sqrt{6}}{3} \xi x u+(1-4 \xi) z^{2} \\
& +2(1-4 \xi) x z+\frac{2 \sqrt{6}}{3} \xi z u+2 \xi \beta u \Omega_{m} \\
& -2 \xi \lambda y^{2} u+(\gamma-1) \Omega_{m},
\end{aligned}
$$

where $\gamma=1+\omega_{m}$ is defined to be the barotropic index. We need to give the junk parameter $s$ in the autonomous form, such that

$$
\begin{aligned}
s= & -\frac{\dot{H}}{H^{2}}=\frac{3}{2}\left(1+\omega_{\text {tot }}\right)=\frac{3}{2}\left[1+(1-4 \xi) x^{2}-y^{2}\right. \\
& +\left(\frac{2}{3} \xi+4 \xi^{2}\right) s u^{2}+\left(8 \xi^{2}-\xi\right) u^{2}+\frac{2 \sqrt{6}}{3} \xi x u \\
& +(1-4 \xi) z^{2}+2(1-4 \xi) x z+\frac{2 \sqrt{6}}{3} \xi z u \\
& \left.+2 \xi \beta u \Omega_{m}-2 \xi \lambda y^{2} u+(\gamma-1) \Omega_{m}\right]
\end{aligned}
$$

Pulling $s$ from the right-hand side of (38) to the left-hand side gives

$$
\begin{aligned}
s= & {\left[1+(1-4 \xi) x^{2}-y^{2}+\left(8 \xi^{2}-\xi\right) u^{2}+\frac{2 \sqrt{6}}{3} \xi x u\right.} \\
& +(1-4 \xi) z^{2}+2(1-4 \xi) x z+\frac{2 \sqrt{6}}{3} \xi z u \\
& \left.+2 \xi \beta u \Omega_{m}-2 \xi \lambda y^{2} u+(\gamma-1) \Omega_{m}\right]\left[\frac{2}{3}-\frac{2}{3} \xi u^{2}\right. \\
& \left.-4 \xi^{2} u^{2}\right]^{-1} .
\end{aligned}
$$


TABLE 1: Critical points and existence conditions.

\begin{tabular}{lcccccc}
\hline Label & $x+z$ & $y$ & $u$ & $\omega_{\text {tot }}$ & $q_{D}$ & Existence \\
\hline$A$ & 0 & 1 & 0 & -1 & -1 & $\lambda=0, \Omega_{m}=0$ \\
$B$ & 0 & -1 & 0 & -1 & -1 & $\lambda=0, \Omega_{m}=0$ \\
$C$ & 0 & $\sqrt{(4 \xi / \lambda)\left(-2 / \lambda+\sqrt{4 / \lambda^{2}+1 / \xi}\right)}$ & $\left(-2 / \lambda+\sqrt{4 / \lambda^{2}+1 / \xi}\right)$ & -1 & -1 & $\lambda \neq 0, \Omega_{m}=0$ \\
$D$ & 0 & $-\sqrt{(4 \xi / \lambda)\left(-2 / \lambda+\sqrt{4 / \lambda^{2}+1 / \xi}\right)}$ & $\left(-2 / \lambda+\sqrt{4 / \lambda^{2}+1 / \xi}\right)$ & -1 & -1 & $\lambda \neq 0, \Omega_{m}=0$ \\
\hline
\end{tabular}

While $s$ is a junk parameter alone, it gains physical meaning in the deceleration parameter $q_{D}$, such that

$$
\begin{aligned}
q_{D} & =-1+s=-1+\left[1+(1-4 \xi) x^{2}-y^{2}\right. \\
& +\left(8 \xi^{2}-\xi\right) u^{2}+\frac{2 \sqrt{6}}{3} \xi x u+(1-4 \xi) z^{2} \\
& +2(1-4 \xi) x z+\frac{2 \sqrt{6}}{3} \xi z u+2 \xi \beta u \Omega_{m}-2 \xi \lambda y^{2} u \\
& \left.+(\gamma-1) \Omega_{m}\right]\left[\frac{2}{3}-\frac{2}{3} \xi u^{2}-4 \xi^{2} u^{2}\right]^{-1} .
\end{aligned}
$$

Now we convert the Friedmann equations (18), the continuity equation (20), and the equation of motion (30) into the autonomous system by using the auxiliary variables in (31) and their derivatives with respect to $N=\ln a$. For any quantity $F$, this derivative has the relation with the time derivative as $\dot{F}=H(d F / d N)=H F^{\prime}$. Then we will obtain $X^{\prime}=f(X)$, where $X$ is the column vector including the auxiliary variables and $f(X)$ is the column vector of the autonomous equations. We then find the critical points $X_{c}$ of $X$, by setting $X^{\prime}=0$. We then expand $X^{\prime}=f(X)$ around $X=X_{c}+U$, where $U$ is the column vector of perturbations of the auxiliary variables, such as $\delta x, \delta y, \delta z$, and $\delta u$ for each constituent in our model. Thus, we expand the perturbation equations up to the first order for each critical point as $U^{\prime}=$ $M U$, where $M$ is the matrix of perturbation equations. The eigenvalues of perturbation matrix $M$ determine the type and stability of each critical point [99-108]. Then the autonomous form of the cosmological system is

$$
\begin{aligned}
x^{\prime}= & -3 x-3 z+s x-z^{\prime}+s z+\sqrt{6} \xi s u-2 \sqrt{6} \xi u \\
& +\frac{\sqrt{6}}{2} \lambda y^{2}-\frac{\sqrt{6}}{2} \beta \Omega_{m}, \\
y^{\prime}= & s y-\frac{\sqrt{6}}{2} \lambda y x-\frac{\sqrt{6}}{2} \lambda y z, \\
z^{\prime}= & -3 x-3 z+s x-x^{\prime}+s z+\sqrt{6} \xi s u-2 \sqrt{6} \xi u \\
& +\frac{\sqrt{6}}{2} \lambda y^{2}-\frac{\sqrt{6}}{2} \beta \Omega_{m}, \\
u^{\prime}= & \sqrt{6} x+\sqrt{6} z .
\end{aligned}
$$

Here (41) and (43) in fact give the same autonomous equations, which means that the variables $x$ and $z$ do not form an orthonormal basis in the phase-space. However, $+z, y$, and $u$ form a complete orthonormal set for the phase-space. Therefore, we set (41) and (43) in a single autonomous equation as

$$
\begin{aligned}
x^{\prime}+z^{\prime}= & -3 x-3 z+s x+s z+\sqrt{6} \xi s u-2 \sqrt{6} \xi u \\
& +\frac{\sqrt{6}}{2} \lambda y^{2}-\frac{\sqrt{6}}{2} \beta \Omega_{m} .
\end{aligned}
$$

The autonomous equation system (42), (44), and (45) represents three invariant submanifolds $+z=0, y=0$, and $u=0$ which, by definition, cannot be intersected by any orbit. This means that there is no global attractor in the deformed dark energy cosmology [109]. We will make finite analysis of the phase space. The finite fixed points are found by setting the derivatives of the invariant submanifolds of the auxiliary variables. We can also write these autonomous equations in $q \rightarrow$ 1 limit in terms of the standard auxiliary variables, such as

$$
\begin{aligned}
x_{s}^{\prime}= & -3 x_{s}+s_{s} x_{s}+\sqrt{6} \xi s_{s} u_{s}-2 \sqrt{6} \xi u_{s}+\frac{\sqrt{6}}{2} \lambda y_{s}^{2} \\
& \quad-\frac{\sqrt{6}}{2} \beta \Omega_{m}, \\
y_{s}^{\prime}= & s_{s} y_{s}-\frac{\sqrt{6}}{2} \lambda y_{s} x_{s}, \\
u_{s}^{\prime}= & \sqrt{6} x_{s} .
\end{aligned}
$$

Here we need to get the finite fixed points (critical points) of the autonomous system in (41)-(45), in order to perform the phase-space analysis of the model. We will obtain these points by equating the left-hand sides of the equations (42), (44), and (45) to zero, by using $\Omega_{\text {tot }}=1$ in (34) and also by assuming $\omega_{\text {tot }}=-1$ and $q_{d}=-1$ in (37) and (40), for each critical point. After some calculations, four sets of solutions are found as the critical points which are listed in Table 1 with the existence conditions. The same critical points are also valid for $x_{s}, y_{s}$, and $u_{s}$ instead of $x+z, y$, and $u$, in the $q \rightarrow 1$ standard dark energy model limit.

Now we should find $\delta s$ from (39), which will exist in the perturbations $\delta x^{\prime}+\delta z^{\prime}, \delta y^{\prime}$, and $\delta u^{\prime}$, such that

$$
\begin{aligned}
\delta s & =\left[2(1-4 \xi)(x+z)+\frac{2 \sqrt{6}}{3} \xi u\right] \frac{1}{P}(\delta x+\delta z) \\
& +[-2 y-4 \xi \lambda y u] \frac{1}{P} \delta y+\left[\left(8 \xi^{2}-\xi\right) 2 u\right.
\end{aligned}
$$




$$
\begin{aligned}
& +\frac{2 \sqrt{6}}{3} \xi(x+z)+2 \xi \beta \Omega_{m}-2 \xi \lambda y^{2} \\
& \left.+\left(\frac{4}{3} \xi+8 \xi^{2}\right) s u\right] \frac{1}{P} \delta u,
\end{aligned}
$$

where $P=2 / 3-(2 / 3) \xi u^{2}-4 \xi^{2} u^{2}$. Then the perturbations $\delta x^{\prime}+\delta z^{\prime}, \delta y^{\prime}$ and $\delta u^{\prime}$ for each phase-space coordinate in our model can be found by using the variations of (42), (44), and (45), such that

$$
\begin{aligned}
& \delta x^{\prime}+\delta z^{\prime}=\left[2(1-4 \xi)(x+z)^{2}+(-s+3) P+4 \xi^{2} u^{2}\right. \\
& \left.+\left(\frac{8}{3} \xi-8 \xi^{2}\right) \sqrt{6}(x+z) u\right] \frac{1}{P}(\delta x+\delta z) \\
& +[(-2(x+z)+\sqrt{6} \lambda P) y-4 \xi \lambda(x+z) y u \\
& \left.-2 \sqrt{6} \xi y u-4 \sqrt{6} \xi^{2} \lambda y u^{2}\right] \frac{1}{P} \delta y \\
& +\left[\left(10 \xi^{2}-\xi\right) 2(x+z) u+\frac{2 \sqrt{6}}{3} \xi(x+z)^{2}\right. \\
& +2 \xi \beta(x+z) \Omega_{m}-2 \xi \lambda(x+z) y^{2} \\
& +\left(\frac{4}{3} \xi+8 \xi^{2}\right)(x+z) s u+\left(8 \xi^{2}-\xi\right) 2 \sqrt{6} \xi u^{2} \\
& +2 \sqrt{6} \xi^{2} \beta u \Omega_{m}-2 \sqrt{6} \xi^{2} \lambda y^{2} u \\
& \left.+\left(\frac{4}{3} \xi+8 \xi^{2}\right) \sqrt{6} \xi s u^{2}+\sqrt{6} \xi(s-2) P\right] \frac{1}{P} \delta u, \\
& \delta y^{\prime}=\left[2(1-4 \xi)(x+z) y+\frac{2 \sqrt{6}}{3} \xi u y-\frac{\sqrt{6}}{2} \lambda y P\right] \\
& \cdot \frac{1}{P}(\delta x+\delta z)+\left[-2 y^{2}-4 \xi \lambda y^{2} u+s P\right. \\
& \left.-\frac{\sqrt{6}}{2} \lambda(x+z) P\right] \frac{1}{P} \delta y+\left[\left(8 \xi^{2}-\xi\right) u y\right. \\
& +\frac{2 \sqrt{6}}{3} \xi(x+z) y+2 \xi \beta y \Omega_{m}-2 \xi \lambda y^{3} \\
& \left.+\left(\frac{4}{3} \xi+8 \xi^{2}\right) s y u\right] \frac{1}{P} \delta u, \\
& \delta u^{\prime}=\sqrt{6}(\delta x+\delta z) .
\end{aligned}
$$

$$
M_{A}=M_{B}=\left(\begin{array}{ccc}
-3 & 0 & -2 \sqrt{6} \xi \\
0 & -3 & 0 \\
\sqrt{6} & 0 & 0
\end{array}\right) \text {, }
$$

From (48), we find the $3 \times 3$ perturbation matrix $M$ whose elements are given as

$$
\begin{aligned}
M_{11} & =\left[2(1-4 \xi)(x+z)^{2}+(-s+3) P+4 \xi^{2} u^{2}\right. \\
+ & \left.\left(\frac{8}{3} \xi-8 \xi^{2}\right) \sqrt{6}(x+z) u\right] \frac{1}{P} \\
M_{12} & =[(-2(x+z)+\sqrt{6} \lambda P) y-4 \xi \lambda(x+z) y u \\
- & \left.2 \sqrt{6} \xi y u-4 \sqrt{6} \xi^{2} \lambda y u^{2}\right] \frac{1}{P}, \\
M_{13} & =\left[\left(10 \xi^{2}-\xi\right) 2(x+z) u+\frac{2 \sqrt{6}}{3} \xi(x+z)^{2}\right. \\
+ & 2 \xi \beta(x+z) \Omega_{m}-2 \xi \lambda(x+z) y^{2} \\
+ & \left(\frac{4}{3} \xi+8 \xi^{2}\right)(x+z) s u+\left(8 \xi^{2}-\xi\right) 2 \sqrt{6} \xi u^{2} \\
M_{32} & =2 \sqrt{6} \xi_{33}^{2} \beta u \Omega_{m}-2 \sqrt{6} \xi^{2} \lambda y^{2} u \\
M_{23} & =\left[\left(8 \xi^{2}-\xi\right) u y+\frac{2 \sqrt{6}}{3} \xi(x+z) y+2 \xi \beta y \Omega m\right. \\
+ & \frac{1}{P}, \\
M_{22} & \left.=\left[-2 y^{2}-4 \xi \lambda y^{2} u-+s P-\frac{4}{3} \lambda+8 \xi^{2}\right) \sqrt{6} \xi s u^{2}+\sqrt{6} \xi(s-2) P\right] \frac{1}{P}, \\
M_{2} & =\left[2(1-4 \xi)(x+z) y+\frac{2 \sqrt{6}}{3} \xi u y-\frac{\sqrt{6}}{2} \lambda y P\right] \\
& =\left[\frac{1}{P},\right.
\end{aligned}
$$

We insert the linear perturbations $(x+z) \rightarrow\left(x_{c}+z_{c}\right)+$ $(\delta x+\delta z), y \rightarrow y_{c}+\delta y$, and $u \rightarrow u_{c}+\delta u$ about the critical points in the autonomous system (42), (44), and (45), in order to calculate the eigenvalues of perturbation matrix $M$ for four critical points given in Table 1, with the corresponding existing conditions. Therefore, we first give the four perturbation matrices for the critical points $A, B, C$, and $D$ with the corresponding existing conditions, such that 


$$
\begin{aligned}
& M_{C} \\
& =\left(\begin{array}{ccc}
\frac{4 \xi^{2} u_{C}^{2}}{P}-3 & -\frac{4 \sqrt{6} \lambda u_{C}^{2} \xi^{2}}{P}-\frac{2 \sqrt{6} \xi y_{C} u_{C}}{P}+\sqrt{6} y_{C} \lambda-\frac{\sqrt{6}\left(2 \xi^{2}-16 \xi^{3}\right) u_{C}^{2}}{P}-\frac{2 \sqrt{6} \xi^{2} \lambda y_{C}^{2} u_{C}}{P}-2 \sqrt{6} \xi \\
\frac{2 \sqrt{6} \xi y_{C} u_{C}}{3 P}-\frac{\sqrt{6} \lambda y_{C}}{2} & -\frac{2 y_{C}^{2}}{P}-\frac{4 \xi \lambda y_{C}^{2} u_{C}}{P} & -\frac{2 \xi \lambda y_{C}^{3}}{P}-\frac{\left(2 \xi-16 \xi^{2}\right) u_{C} y_{C}}{P} \\
\sqrt{6} & 0
\end{array}\right),
\end{aligned}
$$

where $y_{C}=\sqrt{4 \xi / \lambda\left(-2 / \lambda+\sqrt{4 / \lambda^{2}+1 / \xi}\right)}$ and $u_{C}=-2 / \lambda_{+}$ $\sqrt{4 / \lambda^{2}+1 / \xi}$

$$
\begin{aligned}
& M_{D}
\end{aligned}
$$

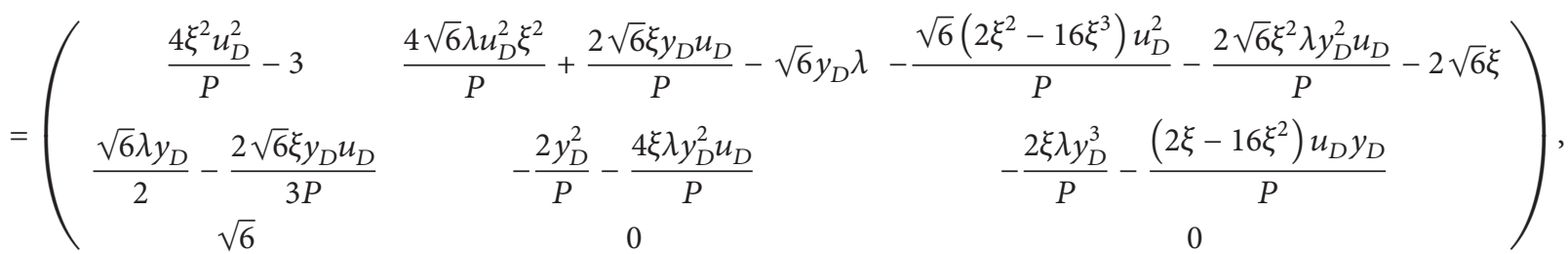

where $y_{D}=-\sqrt{4 \xi / \lambda\left(-2 / \lambda+\sqrt{4 / \lambda^{2}+1 / \xi}\right)}$ and $u_{D}=-2 / \lambda+$ $\sqrt{4 / \lambda^{2}+1 / \xi}$. Also by using $x_{s}, y_{s}$, and $u_{s}$ instead of $x+z, y$, and $u$ in the perturbation matrix elements above, we obtain the standard perturbation matrix elements in $q \rightarrow 1$ limit. Then substituting the standard critical points we again obtain the same matrices $M_{A}, M_{B}, M_{C}$, and $M_{D}$. Therefore the stability of the standard model agrees with the stability of the deformed model.

We need to obtain the four sets of eigenvalues and investigate the sign of the real parts of eigenvalues, so that we can determine the type and stability of critical points. If all the real parts of the eigenvalues are negative, the critical point is said to be stable. The physical meaning of the stable critical point is a stable attractor; namely, the Universe keeps its state forever in this state and thus it can attract the universe at a late time. Here an accelerated expansion phase occurs because $\omega_{\text {tot }}=$ $-1<-1 / 3$. However, if the suitable conditions are satisfied, there can even exist an accelerated contraction for $\omega_{\text {tot }}=$ $-1<-1 / 3$ value. Eigenvalues of the four $M$ matrices and the stability conditions are represented in Table 2 , for each critical point $A, B, C$, and $D$. From Table 2 , the first two critical points $A$ and $B$ have the same eigenvalues, as $C$ and $D$ have the same eigenvalues, too. Here the eigenvalues and the stability conditions of the perturbation matrices for critical points $C$ and $D$ have been obtained by the numerical methods, due to the complexity of the matrices (51) and (52). The stability conditions of each critical point are listed in Table 2, according to the sign of the real part of the eigenvalues.

Now we will study the cosmological behavior of each critical point by considering the attractor solutions in scalar field cosmology [110]. We know that the energy density of a scalar field has a role in the determination of the evolution of
Universe. Cosmological attractors provide the understanding of evolution and the factors affecting on this evolution, such that, from the dynamical conditions, the evolution of scalar field approaches a particular type of behavior without using the initial fine tuning conditions [111-121]. We know that the attractor solutions imply a behavior in which a collection of phase-space points evolve into a particular region and never leave from there. In order to solve the differential equation system (42), (44), and (45) we use adaptive Runge-Kutta method of 4 th and 5th order, in MATLAB programming. We use the present day values for the dark matter density parameter $\Omega_{m}=0.3$, interaction parameter $\beta=14.5$, and $0<\gamma<2$ values in solving the differential equation system $[94,122]$. Then the solutions with the stability conditions of critical points are plotted for each pair of the solution set being the auxiliary variables $x+z, y$, and $u$.

Critical Point $A$. This point exists for $\lambda=0$ which means that the potential $V$ is constant. Acceleration occurs at this point because of $\omega_{\text {tot }}=-1<-1 / 3$, and it is an expansion phase since $y$ is positive, so $H$ is positive, too. Point $A$ is stable meaning that Universe keeps its further evolution, for $0<$ $\xi \leq 3 / 16$ with $\lambda, \beta \in \Re$, but it is a saddle point meaning the universe evolves between different states for $\xi<0$. In Figure 1 , we illustrate the 2 -dimensional projections of 4-dimensional phase-space trajectories for the stability condition $\xi=0.15$ and for the present day values $\beta=14.5, \gamma=1.5$, and $\Omega_{m}=0.3$ and three auxiliary $\lambda$ values. This state corresponds to a stable attractor starting from the critical point $A=(0,1,0)$, as seen from the plots in Figure 1.

Critical Point $B$. Point $B$ also exists for $\lambda=0$ meaning that the potential $V$ is constant. Acceleration phase is again valid here 
TABLE 2: Eigenvalues and stability of critical points.

\begin{tabular}{|c|c|c|c|c|c|c|}
\hline $\begin{array}{l}\text { Critical } \\
\text { points }\end{array}$ & & Eigenvalues & & $\xi$ & $\lambda$ & Stability \\
\hline$A$ and $B$ & -3.0000 & $-(1 / 2) \sqrt{9-48 \xi}-3 / 2$ & $(1 / 2) \sqrt{9-48 \xi}-3 / 2$ & & & $\begin{array}{c}\text { Stable point for } 0<\xi \leq 3 / 16 \\
\text { with } \lambda, \beta \in \Re \\
\text { Saddle point for } \xi<0 \text { with } \\
\lambda, \beta \in \mathfrak{R}\end{array}$ \\
\hline \multirow{11}{*}{$C$ and $D$} & -1.0642 & -1.5576 & -5.5000 & 0.1000 & 1.0000 & \multirow{11}{*}{$\begin{array}{l}\text { Stable point for } 0<\xi, \lambda=1 \text { and } \\
\qquad \beta \in \mathfrak{R} \\
\text { Saddle point, if } \xi<0 \text { and } \lambda \neq 1\end{array}$} \\
\hline & -1.0193 & -1.0193 & -7.2507 & 1.0000 & 1.0000 & \\
\hline & -0.8407 & -0.8407 & -7.7519 & 2.0000 & 1.0000 & \\
\hline & -0.7080 & -0.7080 & -8.0701 & 3.0000 & 1.0000 & \\
\hline & -0.6014 & -0.6014 & -8.3107 & 4.0000 & 1.0000 & \\
\hline & -0.5121 & -0.5121 & -8.5060 & 5.0000 & 1.0000 & \\
\hline & -0.4353 & -0.4353 & -8.6709 & 6.0000 & 1.0000 & \\
\hline & -0.3680 & -0.3680 & -8.8136 & 7.0000 & 1.0000 & \\
\hline & -0.3082 & -0.3082 & -8.9395 & 8.0000 & 1.0000 & \\
\hline & -0.2544 & -0.2544 & -9.0520 & 9.0000 & 1.0000 & \\
\hline & -0.2055 & -0.2055 & -9.1535 & 10.0000 & 1.0000 & \\
\hline
\end{tabular}

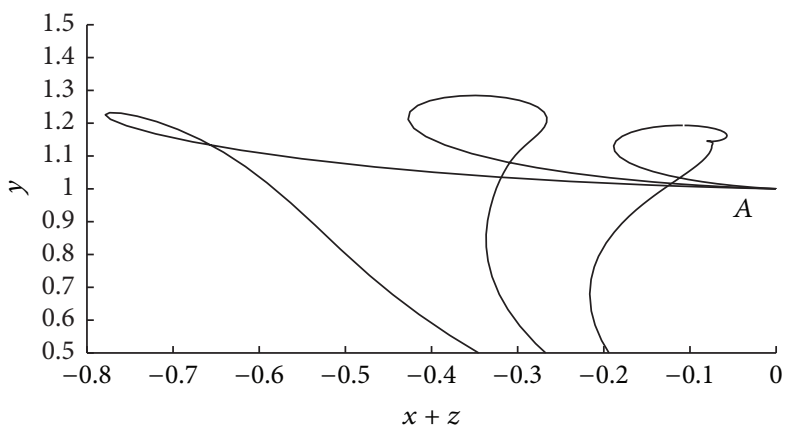

(a)

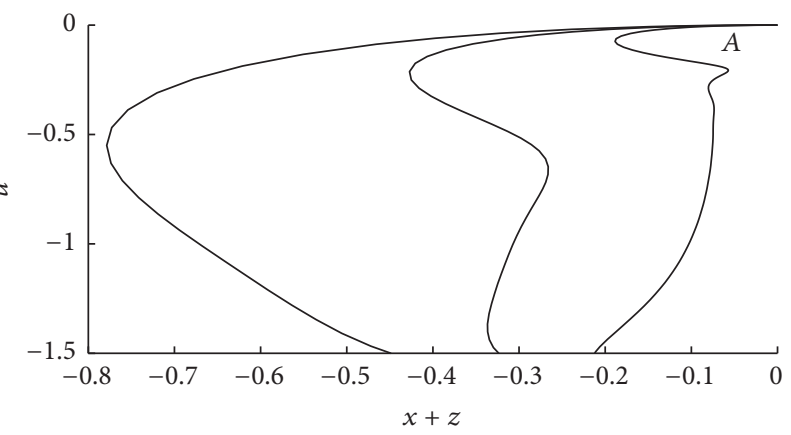

(b)

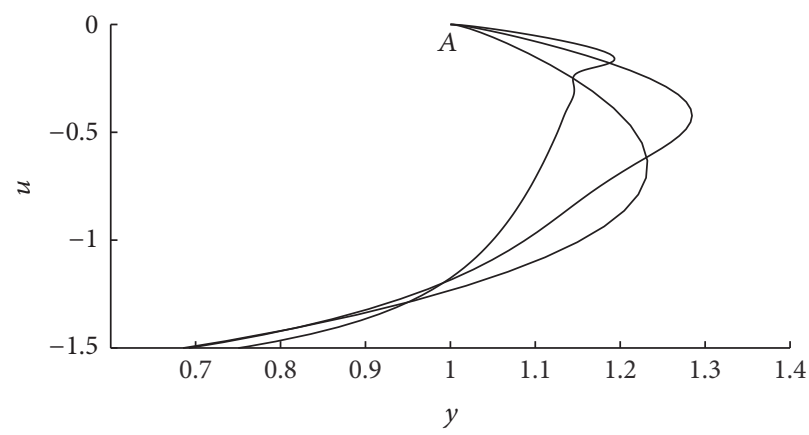

(c)

FIGURE 1: Two-dimensional projections of the phase-space trajectories for stability condition $\xi=0.15$ and for present day values $\beta=14.5$, $\gamma=1.5$, and $\Omega_{m}=0.3$. All plots begin from the critical point $A$ being a stable attractor.

since $\omega_{\text {tot }}=-1<-1 / 3$, but this point refers to contraction phase because $y$ is negative here. For the stability of the point $B$, it is again stable for $0<\xi \leq 3 / 16$ with $\lambda, \beta \in \Re$, but it is a saddle point for $\xi<0$. Therefore the stable attractor behavior is represented for contraction starting from the critical point $B=(0,-1,0)$, as seen from the graphs in Figure 2 . We plot phase-space trajectories for the stability condition $\xi=0.15$ and for the present day values $\beta=14.5, \gamma=1.5$, and $\Omega_{m}=0.3$ and three auxiliary $\lambda$ values.

Critical Point $C$. Critical point $C$ occurs for $\lambda \neq 0$ meaning a field dependent potential $V$. The cosmological behavior is again an acceleration phase since $\omega_{\text {tot }}<-1 / 3$ and an expansion phase since $y$ is positive. Point $C$ is stable for $0<\xi, \lambda=1$, 


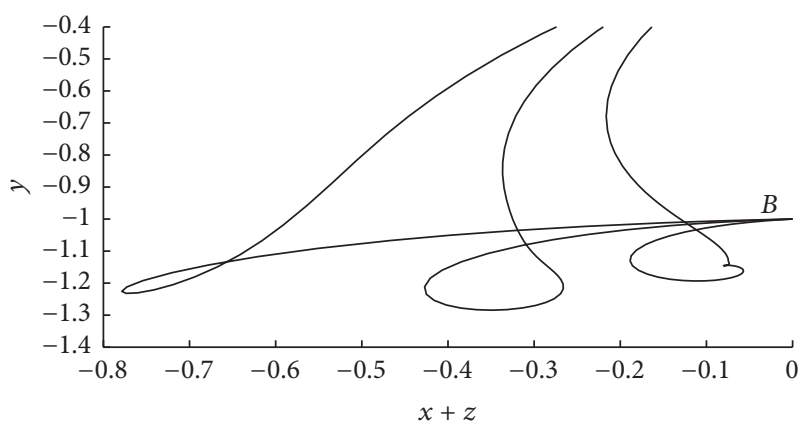

(a)

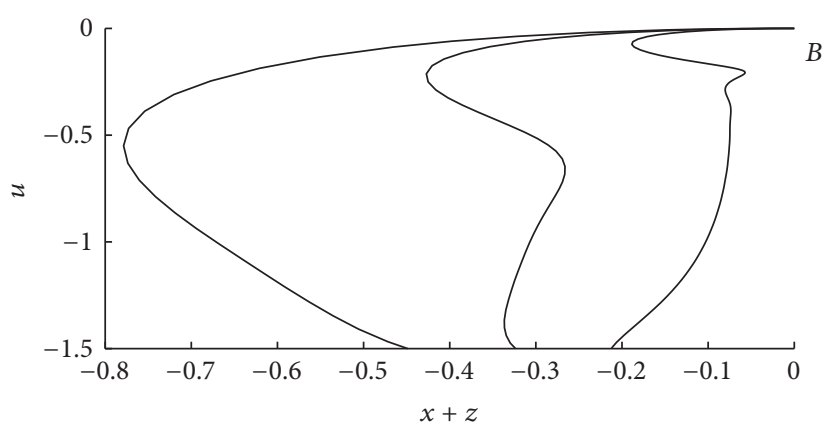

(b)

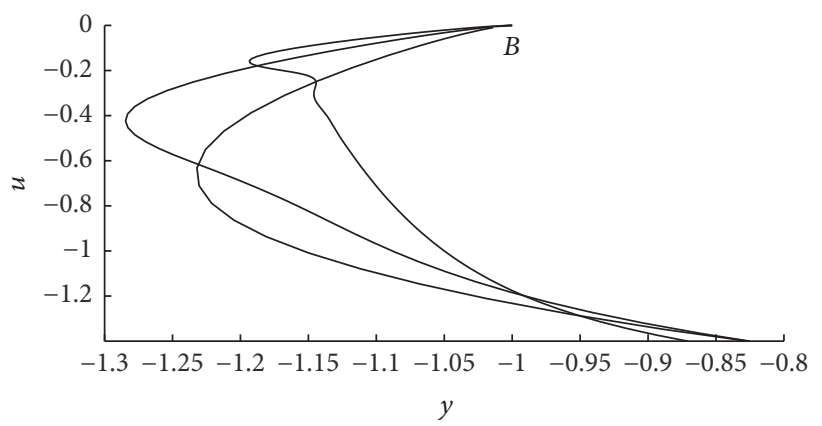

(c)

FIGURE 2: Two-dimensional projections of the phase-space trajectories for stability condition $\xi=0.15$ and for present day values $\beta=14.5$, $\gamma=1.5$, and $\Omega_{m}=0.3$. All plots begin from the critical point $B$ being a stable attractor.

and $\beta \in \Re$ and saddle point if $\xi<0$ and $\lambda \neq 1$.2-dimensional projections of phase-space are represented in Figure 3, for the stability conditions $\xi=2, \lambda=1$ and for the present day values $\beta=14.5, \Omega_{m}=0.3$, and three auxiliary $\gamma$ values in the present day value range. The stable attractor starting from the critical point $C$ can be inferred from the plots in Figure 3 .

Critical Point $D$. This point exists for $\lambda \neq 0$ meaning a field dependent potential $V$. Acceleration phase is again valid due to $\omega_{\text {tot }}<-1 / 3$, but this point refers to a contraction phase because $y$ is negative. Point $D$ is also stable for $0<\xi, \lambda=1$, and $\beta \in \mathfrak{R}$. However, it is a saddle point, while $\xi<0$ and $\lambda \neq 1$. 2-dimensional plots of phase-space trajectories are shown in Figure 4, for the stability conditions $\xi=2, \lambda=1$ and for the present day values $\beta=14.5, \Omega_{m}=0.3$ and three auxiliary $\gamma$ values in the present day value range. This state again corresponds to a stable attractor starting from the point $D$, as seen from the plots in Figure 4 .

All the plots in Figures 1-4 have the structure of stable attractor, since each of them evolves to a single point which is in fact one of the critical points in Table 1. The threedimensional plots of the evolution of phase-space trajectories for the stable attractors are given in Figure 5. These evolutions to the critical points are the attractor solutions of our cosmological model: interacting dark matter and $q$-deformed dark energy nonminimally coupled to gravity, which imply an expanding universe. On the other hand, the construction of the model in the $q \rightarrow 1$ limit reproduces the results of the phase-space analysis for the nondeformed standard dark energy case. The critical points and perturbation matrices are the same for the deformed and standard dark energy models with the equivalence of the auxiliary variables as $x+z=x_{s}$, $y=y_{s}$, and $u=u_{s}$. Therefore, it is confirmed that the dark energy in our model can be defined in terms of the $q$ deformed scalar fields obeying the $q$-deformed boson algebra in (2). According to the stable attractor behaviors, it makes sense to consider the dark energy as a scalar field defined by the $q$-deformed scalar field, with a negative pressure.

We know that the deformed dark energy model is a confirmed model since it reproduces the same stability behaviors, critical points, and perturbation matrices with the standard dark energy model, but the auxiliary variables of deformed and standard models are not the same. The relation between deformed and standard dark energy can be represented regarding auxiliary variable equations in (31):

$$
\begin{aligned}
& x=\sqrt{\frac{1-q^{2 N}}{\left(1-q^{2}\right) N}} x_{s} \\
& y=\sqrt{\exp \left(-c\left(\sqrt{\left.\left.\frac{1-q^{2 N}}{\left(1-q^{2}\right) N}-1\right)\right)} y_{s},\right.\right.} \\
& u=\sqrt{\frac{1-q^{2 N}}{\left(1-q^{2}\right) N}} u_{s}
\end{aligned}
$$

where $c$ is a constant. From the equations (53) we now illustrate the behavior of the deformed and standard dark energy auxiliary variables with respect to the deformation parameter $q$ in Figure 6. We infer from the figure that the value of the 


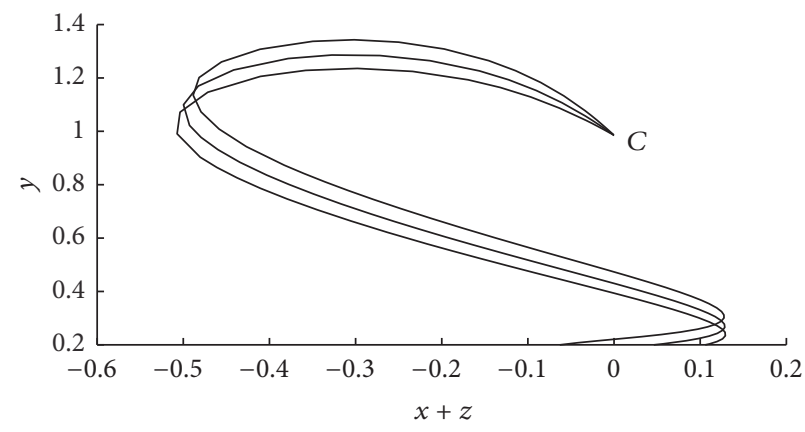

(a)

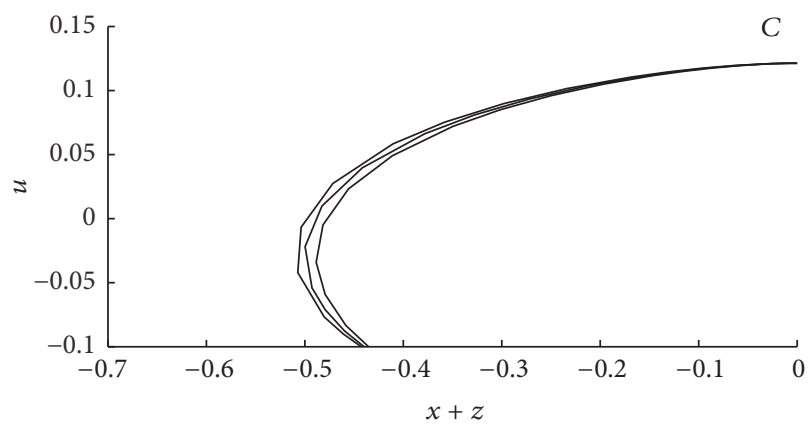

(b)

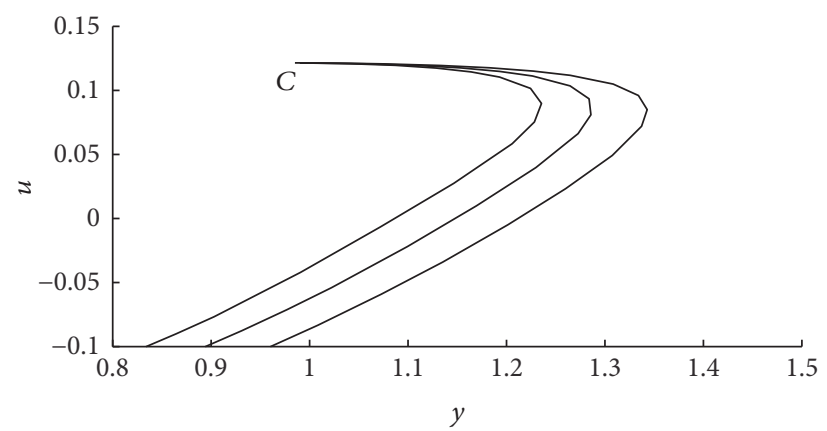

(c)

FIGURE 3: Two-dimensional projections of the phase-space trajectories for stability conditions $\xi=2, \lambda=1$ and for present day values $\beta=14.5$, $\Omega_{m}=0.3$. All plots begin from the critical point $C$ being a stable attractor.

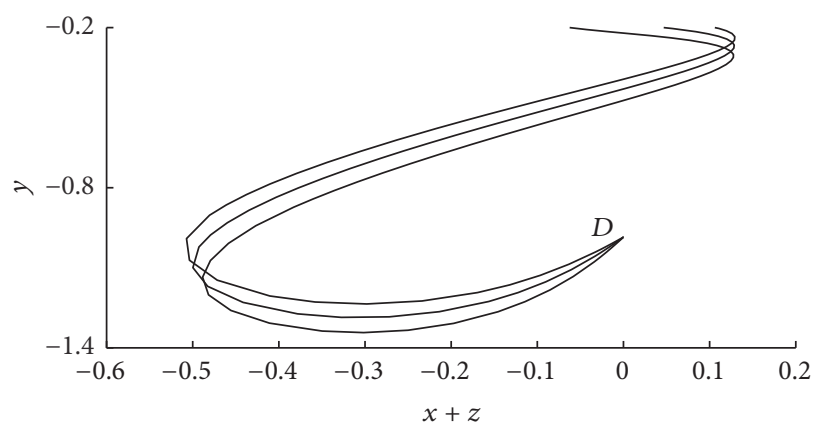

(a)

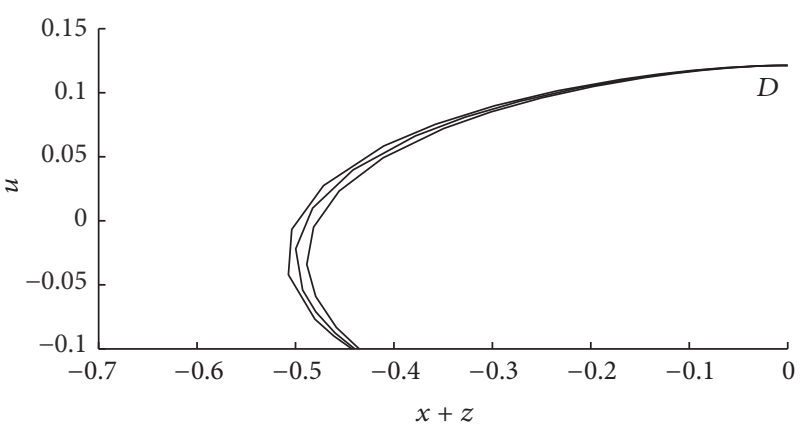

(b)

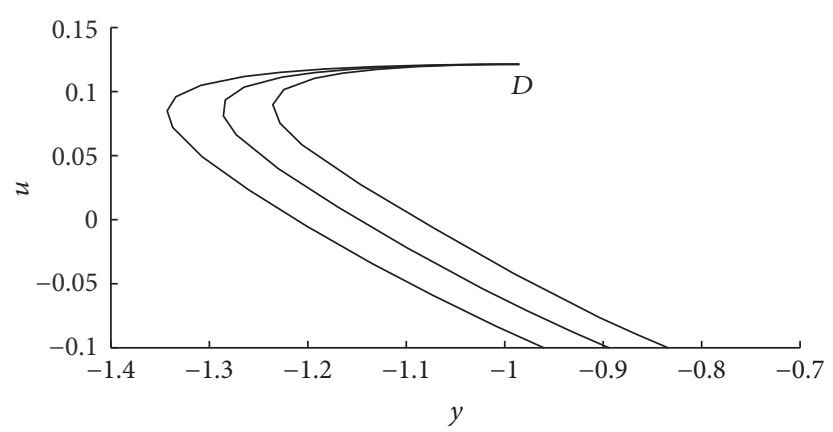

(c)

FIGURE 4: Two-dimensional projections of the phase-space trajectories for stability conditions $\xi=2, \lambda=1$ and for present day values $\beta=14.5$, $\Omega_{m}=0.3$. All plots begin from the critical point $D$ being a stable attractor. 


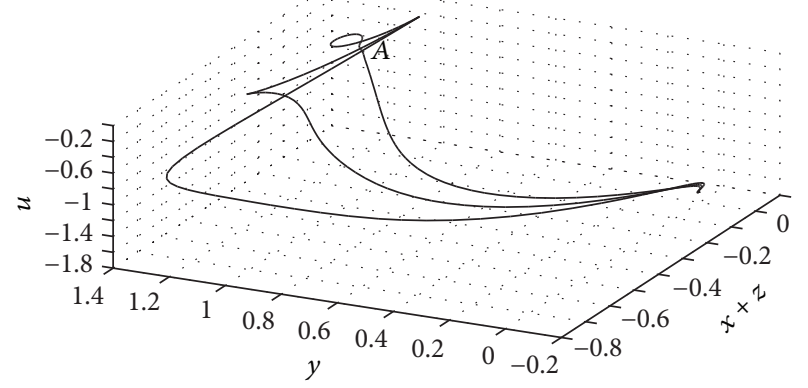

(a)

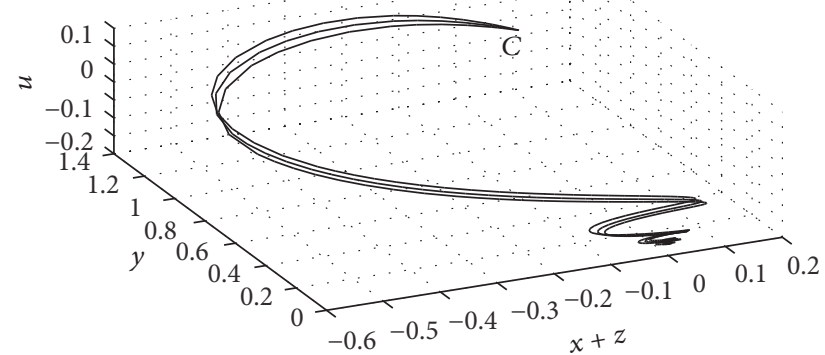

(c)

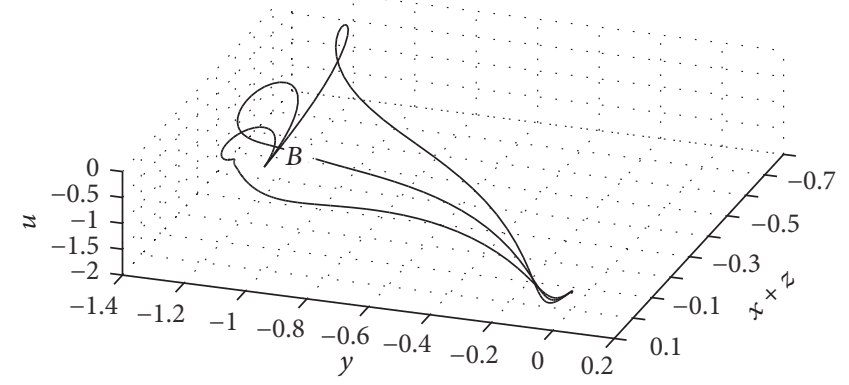

(b)

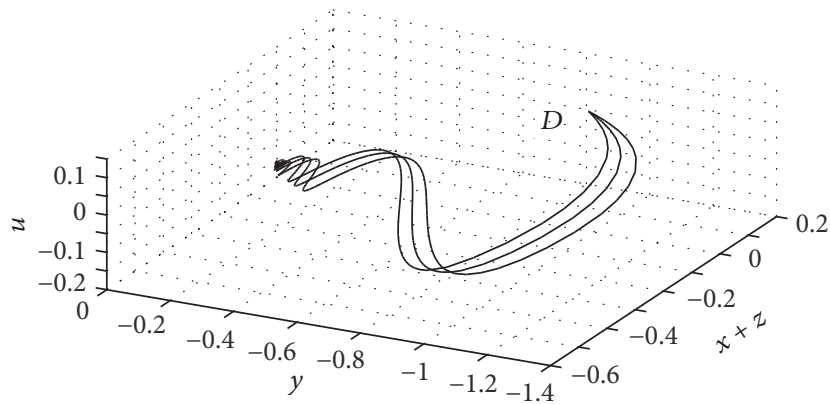

(d)

Figure 5: Three-dimensional plots of the phase-space trajectories for the critical points $A, B, C$, and $D$ being the stable attractors.

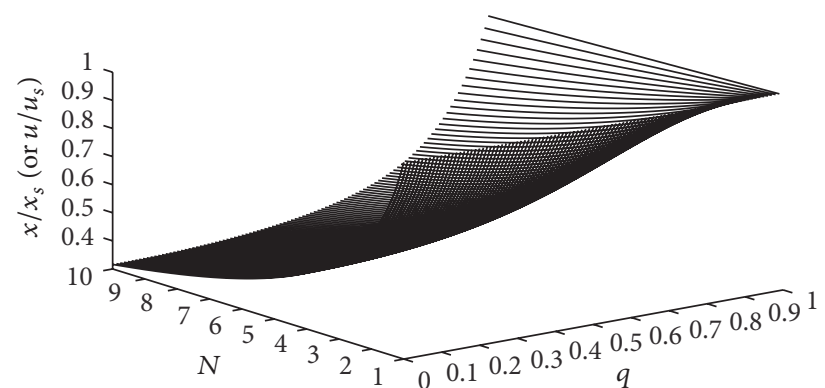

(a)

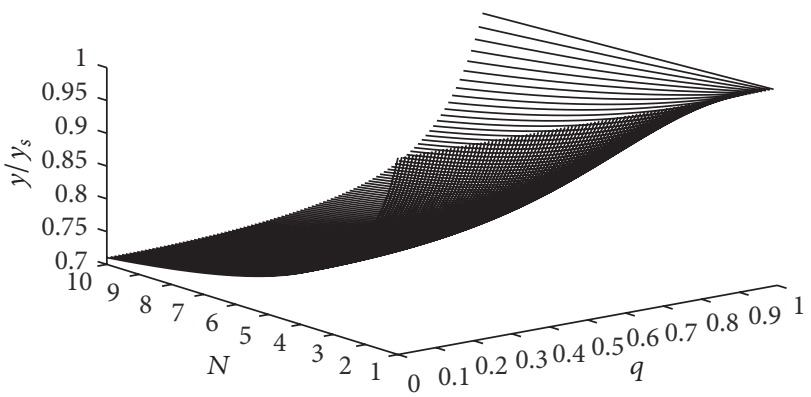

(c)

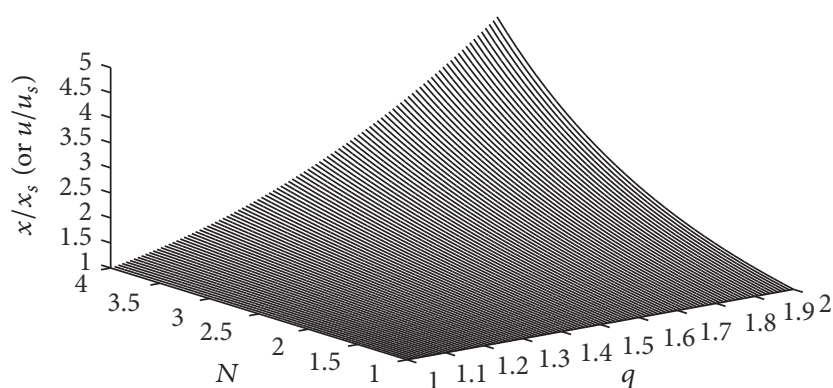

(b)

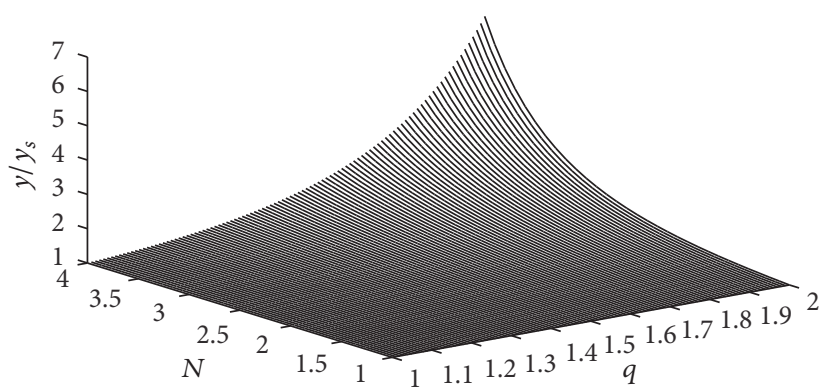

(d)

FIGURE 6: Behavior of the auxiliary variables $x, y$, and $u$ with respect to the deformation parameter $q$ and the particle number $N$. 
deformed $x, y$, and $u$ decreases with decreasing $q$ for the $q<1$ interval for large particle number, and the decrease in the variables $x, y$, and $u$ refers to the decrease in deformed energy density. Also, we conclude that the value of the auxiliary variables $x, y$, and $u$ increases with increasing $q$ for the $q>1$ interval for large particle number. In $q \rightarrow 1$ limit deformed variables goes to standard ones.

\section{Conclusion}

In this study, we propose that the dark energy is formed of the negative-pressure $q$-deformed scalar field whose field equation is defined by the $q$-deformed annihilation and creation operators satisfying the deformed boson algebra in (2), since it is known that the dark energy has a negative pressurelike the deformed bosons-acting as a gravitational repulsion to drive the accelerated expansion of universe. We consider an interacting dark matter and $q$-deformed dark energy nonminimally coupled to the gravity in the framework of Einsteinian gravity in order to confirm our proposal. Then we investigate the dynamics of the model and phase-space analysis whether it will give stable attractor solutions meaning indirectly an accelerating expansion phase of universe. Therefore, we construct the action integral of the interacting dark matter and $q$-deformed dark energy nonminimally coupled to gravity model in order to study its dynamics. With this the Hubble parameter and Friedmann equations of the model are obtained in the spatially flat FRW geometry. Later on, we find the energy density and pressure with the evolution equations for the $q$-deformed dark energy and dark matter from the variation of the action and the Lagrangian of the model. After that we translate these dynamical equations into the autonomous form by introducing the suitable auxiliary variables, in order to perform the phase-space analysis of the model. Then the critical points of autonomous system are obtained by setting each autonomous equation to zero and four perturbation matrices are obtained for each critical point by constructing the perturbation equations. We then determine the eigenvalues of four perturbation matrices to examine the stability of the critical points. We also calculate some important cosmological parameters, such as the total equation of state parameter and the deceleration parameter to check whether the critical points satisfy an accelerating universe. We obtain four stable attractors for the model depending on the coupling parameter $\xi$, interaction parameter $\beta$, and the potential constant $\lambda$. An accelerating universe exists for all stable solutions due to $\omega_{\text {tot }}<-1 / 3$. The critical points $A$ and $B$ are late-time stable attractors for $0<\xi \leq 3 / 16$ and $\lambda, \beta \in \mathfrak{R}$, with the point $A$ referring to an expansion with a stable acceleration, while the point $B$ refers to a contraction. However, the critical points $C$ and $D$ are late-time stable attractors for $0<\xi, \lambda=1$, and $\beta \in \Re$, with the point $C$ referring to an expansion with a stable acceleration, while the point $D$ refers to a contraction. The stable attractor behavior of the model at each critical point is demonstrated in Figures 1-4. In order to solve the differential equation system (42), (44), and (45) with the critical points and plot the graphs in Figures 1-4, we use adaptive Runge-Kutta method of 4 th and 5th order, in
MATLAB programming. Then the solutions with the stability conditions of critical points are plotted for each pair of the solution set being the auxiliary variables in $x+z, y$, and $u$.

These figures show that, by using the convenient parameters of the model according to the existence and stability conditions and the present day values, we can obtain the stable attractors as $A, B, C$, and $D$.

The $q$-deformed dark energy is a generalization of the standard scalar field dark energy. As seen from (9) in the $q \rightarrow 1$ limit, the behavior of the deformed energy density, pressure, and scalar field functions with respect to the standard functions all approach the standard corresponding function values. Consequently, $q$-deformation of the scalar field dark energy gives a self-consistent model due to the existence of standard case parameters of the dark energy in the $q \rightarrow 1$ limit and the existence of the stable attractor behavior of the accelerated expansion phase of universe for the considered interacting and nonminimally coupled dark energy and dark matter model. Although the deformed dark energy model is confirmed through reproducing the same stability behaviors, critical points, and perturbation matrices with the standard dark energy model, the auxiliary variables of deformed and standard models are of course different. By using the auxiliary variable equations in (31), we find the relation between deformed and standard dark energy variables. From these equations, we represent the behavior of the deformed and standard dark energy auxiliary variables with respect to the deformation parameter for $q<1$ and $q>1$ intervals in Figure 6. Then, the value of the deformed $x, y$, and $u$ or equivalently deformed energy density decreases with decreasing $q$ for the $q<1$ interval for large particle number. Also the value of the auxiliary variables $x, y$, and $u$ increases with increasing $q$ for the $q>1$ interval for large particle number. In $q \rightarrow 1$ limit all the deformed variables transform to nondeformed variables.

The consistency of the proposed $q$-deformed scalar field dark energy model is confirmed by the results, since it gives the expected behavior of the universe. The idea of considering the dark energy as a $q$-deformed scalar field is a very recent approach. There are more deformed particle algebras in the literature which can be considered as other and maybe more suitable candidates for the dark energy. As a further study for the confirmation of whether the dark energy can be considered as a general deformed scalar field, the other interactions and couplings between deformed dark energy models, dark matter, and gravity can be investigated in the general relativity framework or in the framework of other modified gravity theories, such as teleparallelism.

\section{Competing Interests}

The author declares that there is no conflict of interests regarding the publication of this paper.

\section{References}

[1] S. Perlmutter, G. Aldering, G. Goldhaber et al., "Measurements of $\Omega$ and $\lambda$ from 42 high-redshift supernovae," The Astrophysical Journal, vol. 517, no. 2, pp. 565-586, 1999. 
[2] A. G. Riess, A. V. Filippenko, P. Challis et al., "Observational evidence from supernovae for an accelerating universe and a cosmological constant," Astronomical Journal, vol. 116, no. 3, pp. 1009-1038, 1998.

[3] U. Seljak, A. Makarov, P. McDonald et al., "Cosmological parameter analysis including SDSS Ly $\alpha$ forest and galaxy bias: constraints on the primordial spectrum of fluctuations, neutrino mass, and dark energy," Physical Review D, vol. 71, no. 10, Article ID 103515, 2005.

[4] M. Tegmark, M. A. Strauss, M. R. Blanton et al., "Cosmological parameters from SDSS andWMAP," Physical Review D, vol. 69, no. 10, Article ID 103501, 2004.

[5] D. J. Eisenstein, I. Zehavi, D. W. Hogg et al., "Detection of the baryon acoustic peak in the large-scale correlation function of SDSS luminous red galaxies," Astrophysical Journal Letters, vol. 633, no. 2, pp. 560-574, 2005.

[6] D. N. Spergel, L. Verde, H. V. Peiris et al., "First-year wilkinson microwave anisotropy probe (WMAP)* observations: determination of cosmological parameters," Astrophysical Journal, Supplement Series, vol. 148, no. 1, pp. 175-194, 2003.

[7] E. Komatsu, K. M. Smith, J. Dunkley et al., "Seven-year wilkinson microwave anisotropy probe (WMAP ${ }^{\star}$ ) observations: cosmological interpretation," The Astrophysical Journal, Supplement Series, vol. 192, no. 2, 2011.

[8] G. Hinshaw, D. Larson, E. Komatsu et al., "Nine-year wilkinson microwave anisotropy probe (WMAP) observations: cosmological parameter results," Astrophysical Journal, Supplement Series, vol. 208, no. 2, article 19, 2013.

[9] P. A. R. Ade, N. Aghanim, C. Armitage-Caplan et al., "Planck 2013 results. XVI. Cosmological parameters," Astronomy \& Astrophysics, vol. 571, article A16, 66 pages, 2013.

[10] P. A. R. Ade, N. Aghanim, M. Arnaud et al., "Planck 2015 results. XIII. Cosmological parameters," Astronomy \& Astrophysics, vol. 594, article A13, 2015.

[11] F. Zwicky, "On the masses of nebulae and of clusters of nebulae," The Astrophysical Journal, vol. 86, pp. 217-246, 1937.

[12] H. W. Babcock, “The rotation of the Andromeda nebula," Lick Observatory Bulletin, vol. 498, pp. 41-51, 1939.

[13] C. Wetterich, "Cosmology and the fate of dilatation symmetry," Nuclear Physics, Section B, vol. 302, no. 4, pp. 668-696, 1988.

[14] B. Ratra and P. J. E. Peebles, "Cosmological consequences of a rolling homogeneous scalar field," Physical Review D, vol. 37, no. 12, p. 3406, 1988.

[15] E. J. Copeland, M. Sami, and S. Tsujikawa, "Dynamics of dark energy," International Journal of Modern Physics. D. Gravitation, Astrophysics, Cosmology, vol. 15, no. 11, pp. 1753-1935, 2006.

[16] M. Li, X.-D. Li, S. Wang, and Y. Wang, "Dark energy," Communications in Theoretical Physics, vol. 56, no. 3, pp. 525-604, 2011.

[17] C. Wetterich, "The cosmon model for an asymptotically vanishing time dependent cosmological 'constant," Astronomy \& Astrophysics, vol. 301, pp. 321-328, 1995.

[18] L. Amendola, "Coupled quintessence," Physical Review D, vol. 62, no. 4, Article ID 043511, 2000.

[19] N. Dalal, K. Abazajian, E. Jenkins, and A. V. Manohar, “Testing the cosmic coincidence problem and the nature of dark energy," Physical Review Letters, vol. 87, no. 14, Article ID 141302, 2001.

[20] W. Zimdahl, D. Pavón, and L. P. Chimento, "Interacting quintessence," Physics Letters, Section B: Nuclear, Elementary Particle and High-Energy Physics, vol. 521, no. 3-4, pp. 133-138, 2001.
[21] B. Gumjudpai, T. Naskar, M. Sami, and S. Tsujikawa, "Coupled dark energy: towards a general description of the dynamics," Journal of Cosmology and Astroparticle Physics, vol. 506, article 7, 2005.

[22] A. P. Billyard and A. A. Coley, "Interactions in scalar field cosmology," Physical Review D, vol. 61, no. 8, Article ID 083503, 2000.

[23] J.-Q. Xia, Y.-F. Cai, T.-T. Qiu, G.-B. Zhao, and X. Zhang, "Constraints on the sound speed of dynamical dark energy," International Journal of Modern Physics D, vol. 17, no. 8, pp. 1229-1243, 2008.

[24] A. Vikman, "Can dark energy evolve to the phantom?" Physical Review D, vol. 71, no. 2, Article ID 023515, 2005.

[25] W. Hu, "Crossing the phantom divide: dark energy internal degrees of freedom," Physical Review D-Particles, Fields, Gravitation and Cosmology, vol. 71, no. 4, Article ID 047301, 2005.

[26] R. R. Caldwell and M. Doran, "Dark-energy evolution across the cosmological-constant boundary," Physical Review DParticles, Fields, Gravitation and Cosmology, vol. 72, no. 4, Article ID 043527, pp. 1-6, 2005.

[27] G.-B. Zhao, J.-Q. Xia, M. Li, B. Feng, and X. Zhang, "Perturbations of the quintom models of dark energy and the effects on observations," Physical Review D, vol. 72, no. 12, Article ID 123515, 2005.

[28] M. Kunz and D. Sapone, "Crossing the phantom divide," Physical Review D, vol. 74, no. 12, Article ID 123503, 2006.

[29] B. L. Spokoiny, "Inflation and generation of perturbations in broken-symmetric theory of gravity," Physics Letters B, vol. 147, no. 1-3, pp. 39-43, 1984.

[30] F. Perrotta, C. Baccigalupi, and S. Matarrese, "Extended quintessence," Physical Review D, vol. 61, no. 2, Article ID 023507, 2000.

[31] E. Elizalde, S. Nojiri, and S. D. Odintsov, "Late-time cosmology in a (phantom) scalar-tensor theory: dark energy and the cosmic speed-up," Physical Review D, vol. 70, no. 4, Article ID 043539, 2004.

[32] K. Bamba, S. Capozziello, S. Nojiri, and S. D. Odintsov, "Dark energy cosmology: the equivalent description via different theoretical models and cosmography tests," Astrophysics and Space Science, vol. 342, no. 1, pp. 155-228, 2012.

[33] O. Hrycyna and M. Szydowski, "Non-minimally coupled scalar field cosmology on the phase plane," Journal of Cosmology and Astroparticle Physics, vol. 2009, no. 4, article no. 26, 2009.

[34] O. Hrycyna and M. Szydłowski, "Extended quintessence with nonminimally coupled phantom scalar field," Physical Review $D$, vol. 76, no. 12, Article ID 123510, 2007.

[35] R. C. de Souza and G. M. Kremer, "Constraining non-minimally coupled tachyon fields by the Noether symmetry," Classical and Quantum Gravity, vol. 26, no. 13, Article ID 135008, 2009.

[36] A. A. Sen and N. C. Devi, "Cosmology with non-minimally coupled k-field," General Relativity and Gravitation, vol. 42, no. 4, pp. 821-838, 2010.

[37] E. Dil and E. Kolay, "Dynamics of mixed dark energy domination in teleparallel gravity and phase-space analysis," Advances in High Energy Physics, vol. 2015, Article ID 608252, 20 pages, 2015.

[38] S. Weinberg, “The cosmological constant problem," Reviews of Modern Physics, vol. 61, no. 1, pp. 1-23, 1989.

[39] S. M. Carroll, W. H. Press, and E. L. Turner, “The cosmological constant," Annual Review of Astronomy and Astrophysics, vol. 30, no. 1, pp. 499-542, 1992. 
[40] L. M. Krauss and M. S. Turner, "The cosmological constant is back," General Relativity and Gravitation, vol. 27, no. 11, pp. 11371144, 1995.

[41] G. Huey, L. Wang, R. Dave, R. R. Caldwell, and P. J. Steinhardt, "Resolving the cosmological missing energy problem," Physical Review D - Particles, Fields, Gravitation and Cosmology, vol. 59, no. 6, pp. 1-6, 1999.

[42] P. Kulish and N. Reshetiknin, "Quantum linear problem for the Sine-Gordon equation and higher representations," Journal of Soviet Mathematics, vol. 23, no. 4, pp. 2435-2441, 1981.

[43] E. Sklyanin, L. Takhatajan, and L. Faddeev, "Quantum inverse problem method. I," Theoretical and Mathematical Physics, vol. 40, no. 2, pp. 688-706, 1979.

[44] L. C. Biedenharn and M. A. Lohe, Quantum Group Symmetry and Q-Tensor Algebras, World Scientific Publishing, River Edge, NJ, USA, 1995.

[45] M. Arik and D. D. Coon, "Hilbert spaces of analytic functions and generalized coherent states," Journal of Mathematical Physics, vol. 17, no. 4, pp. 524-527, 1976.

[46] A. J. Macfarlane, "On q-analogues of the quantum harmonic oscillator and the quantum group SUq(2)," Journal of Physics A, vol. 22, no. 21, pp. 4581-4588, 1989.

[47] L. C. Biedenharn, "The quantum group $\mathrm{SU}_{q}(2)$ and a q-analogue of the boson operators," Journal of Physics. A: Mathematical and General, vol. 22, no. 18, pp. L873-L878, 1989.

[48] L. Tripodi and C. L. Lima, "On a $q$-covariant form of the BCS approximation," Physics Letters. B, vol. 412, no. 1-2, pp. 7-13, 1997.

[49] V. S. Timóteo and C. L. Lima, "Effect of $q$-deformation in the NJL gap equation," Physics Letters B, vol. 448, no. 1-2, pp. 1-5, 1999.

[50] C. R. Lee and J. P. Yu, "On $q$-analogues of the statistical distribution," Physics Letters. A, vol. 150, no. 2, pp. 63-66, 1990.

[51] J. A. Tuszynski, J. L. Rubin, J. Meyer, and M. Kibler, "Statistical mechanics of a q-deformed boson gas," Physics Letters A, vol. 175, no. 3-4, pp. 173-177, 1993.

[52] P. N. Swamy, "Interpolating statistics and $q$-deformed oscillator algebras," International Journal of Modern Physics B, vol. 20, no. 6, pp. 697-713, 2006.

[53] M. R. Ubriaco, "Thermodynamics of a free $\mathrm{SU}_{q}(2)$ fermionic system," Physics Letters. A, vol. 219, no. 3-4, pp. 205-211, 1996.

[54] M. R. Ubriaco, "High and low temperature behavior of a quantum group fermion gas," Modern Physics Letters A, vol. 11, no. 29, pp. 2325-2333, 1996.

[55] M. R. Ubriaco, "Anyonic behavior of quantum group gases," Physical Review E. Statistical, Nonlinear, and Soft Matter Physics, vol. 55, no. 1, part A, pp. 291-296, 1997.

[56] A. Lavagno and P. N. Swamy, "Thermostatistics of a q-deformed boson gas," Physical Review E, vol. 61, no. 2, pp. 1218-1226, 2000.

[57] A. Lavagno and P. N. Swamy, "Generalized thermodynamics of $q$-deformed bosons and fermions," Physical Review EStatistical, Nonlinear, and Soft Matter Physics, vol. 65, no. 3, Article ID 036101, 2002.

[58] A. Algin and M. Baser, "Thermostatistical properties of a twoparameter generalised quantum group fermion gas," Physica A: Statistical Mechanics and Its Applications, vol. 387, no. 5-6, pp. 1088-1098, 2008.

[59] A. Algin, M. Arik, and A. S. Arikan, "High temperature behavior of a two-parameter deformed quantum group fermion gas," Physical Review E, vol. 65, no. 2, Article ID 026140, p. 026140/5, 2002.
[60] A. Algin, A. S. Arikan, and E. Dil, "High temperature thermostatistics of fermionic Fibonacci oscillators with intermediate statistics," Physica A: Statistical Mechanics and its Applications, vol. 416, pp. 499-517, 2014.

[61] O. W. Greenberg, "Particles with small violations of Fermi or Bose statistics," Physical Review D, vol. 43, no. 12, pp. 4111-4120, 1991.

[62] A. M. Gavrilik, I. I. Kachurik, and Y. A. Mishchenko, "Quasibosons composed of two $q$-fermions: realization by deformed oscillators," Journal of Physics A: Mathematical and Theoretical, vol. 44, no. 47, Article ID 475303, 2011.

[63] P. Pouliot, "Finite number of states, de Sitter space and quantum groups at roots of unity," Classical and Quantum Gravity, vol. 21, no. 1, pp. 145-162, 2004.

[64] A. Strominger, "Black hole statistics," Physical Review Letters, vol. 71, no. 21, pp. 3397-3400, 1993.

[65] E. Dil, “Q-deformed einstein equations," Canadian Journal of Physics, vol. 93, no. 11, pp. 1274-1278, 2015.

[66] E. Dil and E. Kolay, "Solution of deformed Einstein equations and quantum black holes," Advances in High Energy Physics, vol. 2016, Article ID 3973706, 7 pages, 2016.

[67] D. Bonatsos, E. N. Argyres, and P. P. Raychev, "SU $\mathrm{S}_{q}(1,1)$ description of vibrational molecular spectra," Journal of Physics A: Mathematical and General, vol. 24, no. 8, pp. L403-L408, 1991.

[68] D. Bonatsos, P. P. Raychev, and A. Faessler, "Quantum algebraic description of vibrational molecular spectra," Chemical Physics Letters, vol. 178, no. 2-3, pp. 221-226, 1991.

[69] D. Bonatsos and C. Daskaloyannis, "Generalized deformed oscillators for vibrational spectra of diatomic molecules," Physical Review A, vol. 46, no. 1, pp. 75-80, 1992.

[70] P. P. Raychev, R. P. Roussev, and Y. F. Smirnov, "The quantum algebra $\mathrm{SU}_{\mathrm{q}}(2)$ and rotational spectra of deformed nuclei," Journal of Physics G: Nuclear and Particle Physics, vol. 16, no. 8, pp. L137-L141, 1990.

[71] A. I. Georgieva, K. D. Sviratcheva, M. I. Ivanov, and J. P. Draayer, " $q$-Deformation of symplectic dynamical symmetries in algebraic models of nuclear structure," Physics of Atomic Nuclei, vol. 74, no. 6, pp. 884-892, 2011.

[72] M. R-Monteiro, L. M. C. S. Rodrigues, and S. Wulck, "Quantum algebraic nature of the phonon spectrum in4He," Physical Review Letters, vol. 76, no. 7, 1996.

[73] A. E. Shalyt-Margolin, "Deformed quantum field theory, thermodynamics at low and high energies, and gravity. II. Deformation parameter," International Journal of Theoretical and Mathematical Physics, vol. 2, no. 3, pp. 41-50, 2012.

[74] A. E. Shalyt-Margolin and V. I. Strazhev, "Dark energy and deformed quantum theory in physics of the early universe. In non-eucleden geometry in modern physics," in Proceedings of the 5th Intentional Conference of Bolyai-Gauss-Lobachevsky (BGL-5 '07), Y. Kurochkin and V. Red'kov, Eds., pp. 173-178, Minsk, Belarus, 2007.

[75] Y. J. Ng, "Holographic foam, dark energy and infinite statistics," Physics Letters, Section B: Nuclear, Elementary Particle and HighEnergy Physics, vol. 657, no. 1-3, pp. 10-14, 2007.

[76] Y. J. Ng, "Spacetime foam," International Journal of Modern Physics D, vol. 11, no. 10, pp. 1585-1590, 2002.

[77] M. Chaichian, R. G. Felipe, and C. Montonen, "Statistics of $q$ oscillators, quons and relations to fractional statistics," Journal of Physics A: Mathematical and General, vol. 26, no. 16, pp. 40174034, 1993. 
[78] J. Wess and B. Zumino, "Covariant differential calculus on the quantum hyperplane," Nuclear Physics B-Proceedings Supplements, vol. 18, no. 2, pp. 302-312, 1991.

[79] G. Vinod, Studies in quantum oscillators and q-deformed quantum mechanics [Ph.D. thesis], Cochin University of Science and Technology, Department of Physics, Kochi, India, 1997.

[80] B. K. Berger, "Scalar particle creation in an anisotropic universe," Physical Review D, vol. 12, no. 2, pp. 368-375, 1975.

[81] S. W. Hawking, "Breakdown of predictability in gravitational collapse," Physical Review D, vol. 14, no. 10, pp. 2460-2473, 1976.

[82] B. K. Berger, "Classical analog of cosmological particle creation," Physical Review D, vol. 18, no. 12, pp. 4367-4372, 1978.

[83] L. Parker, "Quantized fields and particle creation in expanding universes. I," Physical Review, vol. 183, no. 5, article 1057, 1969.

[84] J. W. Goodison and D. J. Toms, "No generalized statistics from dynamics in curved spacetime," Physical Review Letters, vol. 71, no. 20, 1993.

[85] D. Bonatsos and C. Daskaloyannis, "Quantum groups and their applications in nuclear physics," Progress in Particle and Nuclear Physics, vol. 43, no. 1, pp. 537-618, 1999.

[86] R. G. Leigh, "Dirac-born-infeld action from dirichlet $\sigma$-model," Modern Physics Letters A, vol. 04, no. 28, pp. 2767-2772, 1989.

[87] N. A. Chernikov and E. A. Tagirov, "Quantum theory of scalar field in de Sitter space-time," Annales de l'I.H.P. Physique Théorique, vol. 9, pp. 109-141, 1968.

[88] C. G. Callan Jr., S. Coleman, and R. Jackiw, "A new improved energy-momentum tensor," Annals of Physics, vol. 59, pp. 4273, 1970.

[89] V. Faraoni, "Inflation and quintessence with nonminimal coupling," Physical Review D, vol. 62, no. 2, Article ID 023504, 2000.

[90] S. C. Park and S. Yamaguchi, "Inflation by non-minimal coupling," Journal of Cosmology and Astroparticle Physics, vol. 2008, no. 8, p. 9, 2008.

[91] A. de Felice and S. Tsujikawa, " $\mathrm{f}(\mathrm{R})$ theories," Living Reviews in Relativity, vol. 13, article no. 3, 2010.

[92] S. Nojiri and S. D. Odintsov, "Unified cosmic history in modified gravity: from $\mathrm{F}(\mathrm{R})$ theory to Lorentz non-invariant models," Physics Reports, vol. 505, no. 2-4, pp. 59-144, 2011.

[93] S. Nojiri and S. D. Odintsov, "Introduction to modified gravity and gravitational alternative for dark energy," International Journal of Geometric Methods in Modern Physics, vol. 4, no. 1, pp. 115-146, 2007.

[94] A. Banijamali, "Dynamics of interacting tachyonic teleparallel dark energy," Advances in High Energy Physics, vol. 2014, Article ID 631630, 14 pages, 2014.

[95] B. Fazlpour and A. Banijamali, "Tachyonic teleparallel dark energy in phase space," Advances in High Energy Physics, vol. 2013, Article ID 279768, 9 pages, 2013.

[96] P. G. Ferreira and M. Joyce, "Structure formation with a selftuning scalar field," Physical Review Letters, vol. 79, no. 24, pp. 4740-4743, 1997.

[97] E. J. Copeland, A. R. Liddle, and D. Wands, "Exponential potentials and cosmological scaling solutions," Physical Review $D$, vol. 57, no. 8, pp. 4686-4690, 1998.

[98] X.-M. Chen, Y. Gong, and E. N. Saridakis, "Phase-space analysis of interacting phantom cosmology," Journal of Cosmology and Astroparticle Physics, vol. 2009, no. 4, article no. 001, 2009.

[99] Z.-K. Guo, Y.-S. Piao, X. Zhang, and Y.-Z. Zhang, "Cosmological evolution of a quintom model of dark energy," Physics Letters, Section B: Nuclear, Elementary Particle and High-Energy Physics, vol. 608, no. 3-4, pp. 177-182, 2005.
[100] R. Lazkoz and G. León, "Quintom cosmologies admitting either tracking or phantom attractors," Physics Letters B, vol. 638, no. 4, pp. 303-309, 2006.

[101] Z.-K. Guo, Y.-S. Piao, X. Zhang, and Y.-Z. Zhang, “Two-field quintom models in the $w-w^{\prime}$ plane," Physical Review $D$, vol. 74, no. 12, Article ID 127304, 4 pages, 2006.

[102] R. Lazkoz, G. León, and I. Quiros, "Quintom cosmologies with arbitrary potentials," Physics Letters B, vol. 649, no. 2-3, pp. 103110, 2007.

[103] M. Alimohammadi, "Asymptotic behavior of $\omega$ in general quintommodel," General Relativity and Gravitation, vol. 40, no. 1, pp. 107-115, 2008.

[104] M. R. Setare and E. N. Saridakis, "Coupled oscillators as models of quintom dark energy," Physics Letters, Section B, vol. 668, no. 3, pp. 177-181, 2008.

[105] M. R. Setare and E. N. Saridakis, "Quintom cosmology with general potentials," International Journal of Modern Physics. D. Gravitation, Astrophysics, Cosmology, vol. 18, no. 4, pp. 549-557, 2009.

[106] M. R. Setare and E. N. Saridakis, "The quintom model with $\mathrm{O}(\mathrm{N})$ symmetry," Journal of Cosmology and Astroparticle Physics, vol. 2008, no. 9, article no. 026, 2008.

[107] M. R. Setare and E. N. Saridakis, "Quintom dark energy models with nearly flat potentials," Physical Review D, vol. 79, no. 4, Article ID 043005, 2009.

[108] G. Leon, R. Cardenas, and J. L. Morales, "Equilibrium sets in quintom cosmologies: the past asymptotic dynamics," https://arxiv.org/abs/0812.0830.

[109] S. Carloni, E. Elizalde, and P. J. Silva, "An analysis of the phase space of Hořava-Lifshitz cosmologies," Classical and Quantum Gravity, vol. 27, no. 4, Article ID 045004, 2010.

[110] Z.-K. Guo, Y.-S. Piao, R.-G. Cai, and Y.-Z. Zhang, "Inflationary attractor from tachyonic matter," Physical Review D, vol. 68, no. 4, Article ID 043508, 2003.

[111] L. A. Ureña-López and M. J. Reyes-Ibarra, "On the dynamics of a quadratic scalar field potential," International Journal of Modern Physics D, vol. 18, no. 4, pp. 621-634, 2009.

[112] V. A. Belinsky, L. P. Grishchuk, I. M. Khalatnikov, and Y. B. Zeldovich, "Inflationary stages in cosmological models with a scalar field," Physics Letters B, vol. 155, no. 4, pp. 232-236, 1985.

[113] T. Piran and R. M. Williams, "Inflation in universes with a massive scalar field," Physics Letters B, vol. 163, no. 5-6, pp. 331335, 1985.

[114] A. R. Liddle and D. H. Lyth, Cosmological inflation and large-scale structure, Cambridge University Press, Cambridge, England, 2000.

[115] V. V. Kiselev and S. A. Timofeev, "Quasiattractor dynamics of $\lambda \phi 4$-inflation,” https://arxiv.org/abs/0801.2453.

[116] S. Downes, B. Dutta, and K. Sinha, "Attractors, universality, and inflation," Physical Review D, vol. 86, no. 10, Article ID 103509, 2012.

[117] J. Khoury and P. J. Steinhardt, "Generating scale-invariant perturbations from rapidly-evolving equation of state," Physical Review D-Particles, Fields, Gravitation and Cosmology, vol. 83, no. 12, Article ID 123502, 2011.

[118] S. Clesse, C. Ringeval, and J. Rocher, "Fractal initial conditions and natural parameter values in hybrid inflation," Physical Review D, vol. 80, no. 12, Article ID 123534, 2009.

[119] V. V. Kiselev and S. A. Timofeev, "Quasiattractor in models of new and chaotic inflation," General Relativity and Gravitation, vol. 42, no. 1, pp. 183-197, 2010. 
[120] G. F. R. Ellis, R. Maartens, and M. A. H. MacCallum, Relativistic cosmology, Cambridge University Press, Cambridge, UK, 2012.

[121] Y. Wang, J. M. Kratochvil, A. Linde, and M. Shmakova, "Current observational constraints on cosmic doomsday," Journal of Cosmology and Astroparticle Physics, vol. 412, article 6, 2004.

[122] L. Amendola and D. Tocchini-Valentini, "Stationary dark energy: the present universe as a global attractor," Physical Review D, vol. 64, no. 4, Article ID 043509, 2001. 

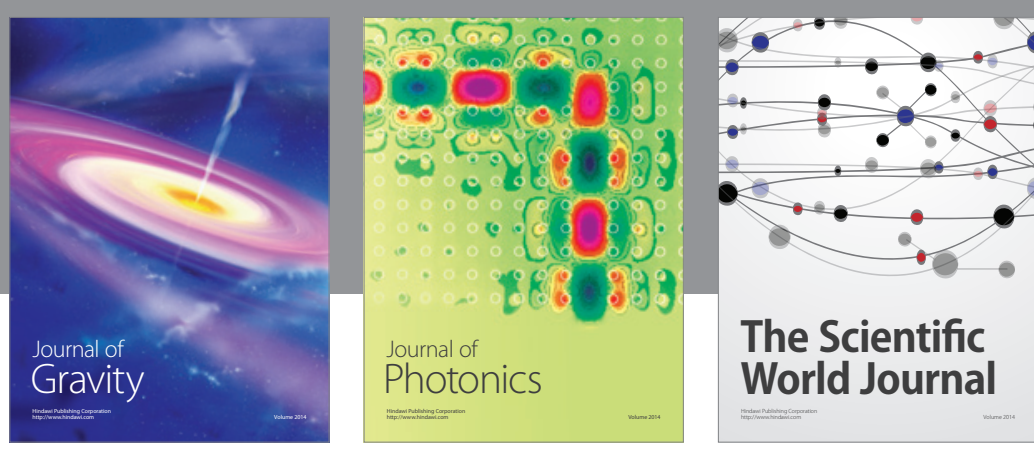

The Scientific World Journal
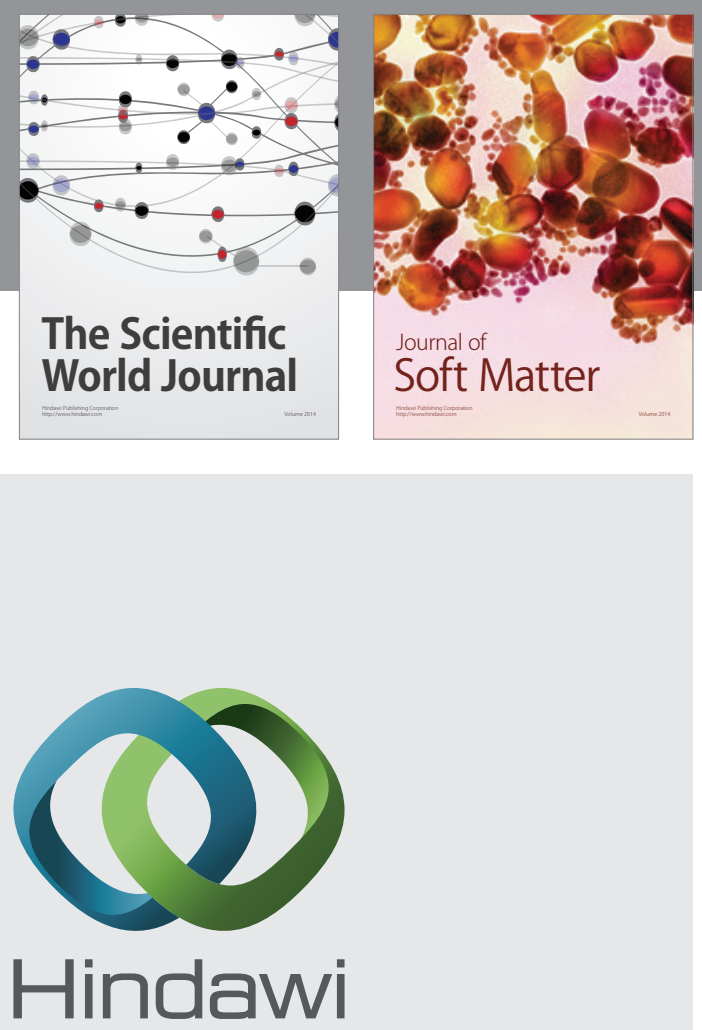

Submit your manuscripts at

http://www.hindawi.com

nternational Journal of

Statistical Mechanics
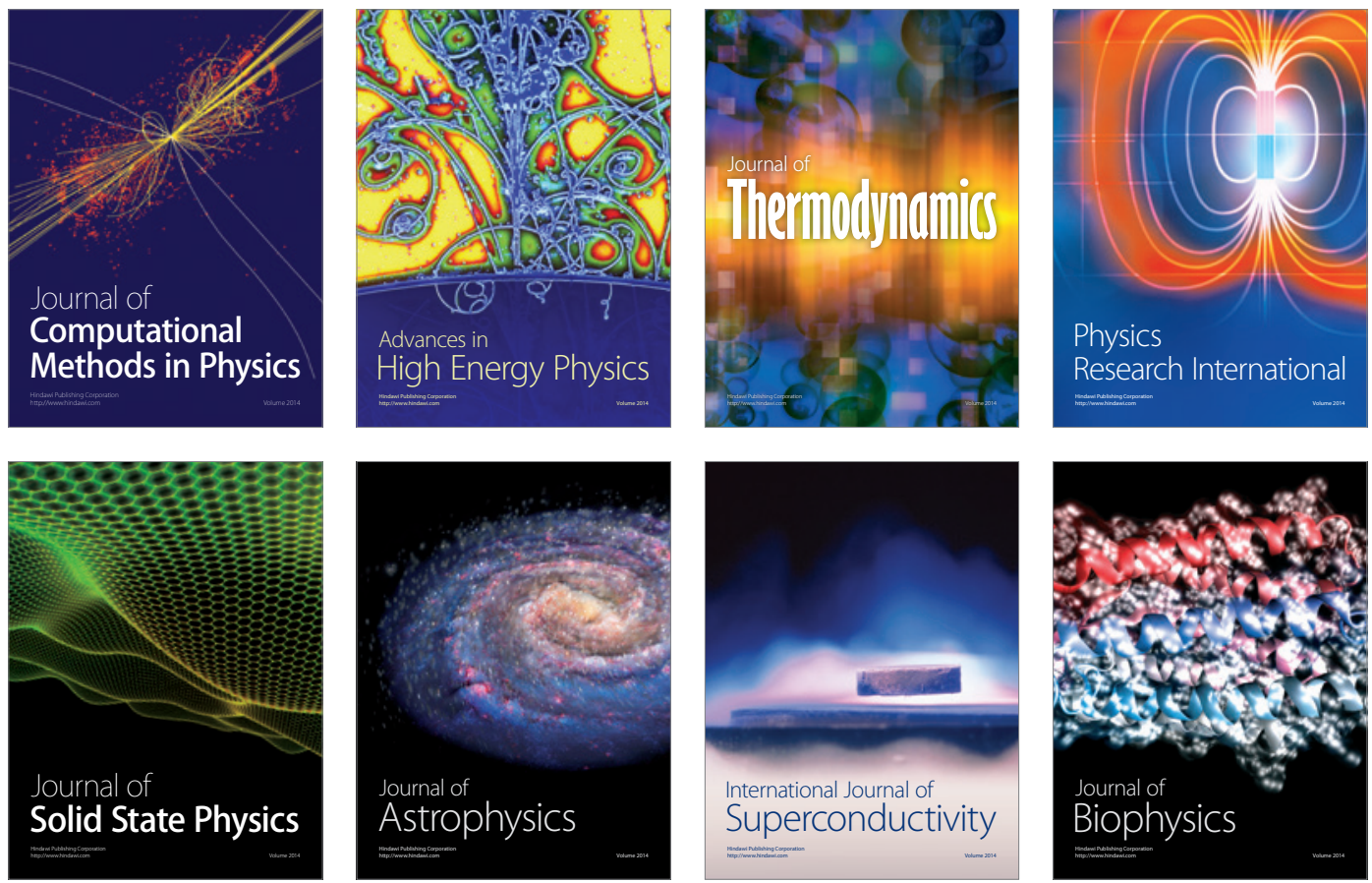
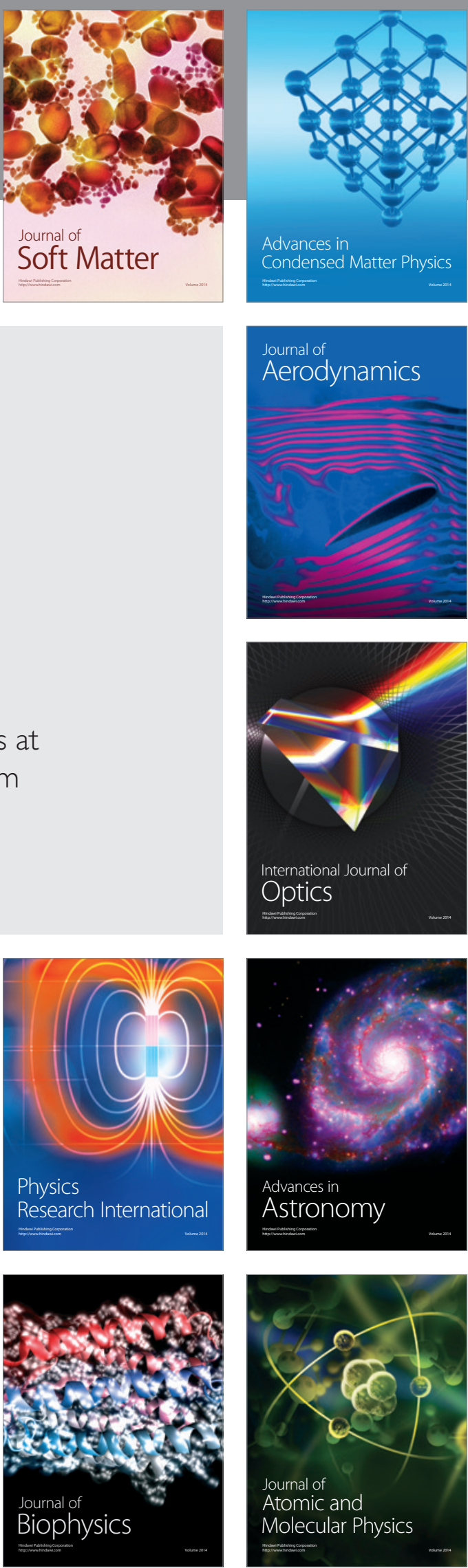